\title{
Numerical and experimental study of a double jet inclination variation on its dynamic evolution within a crossflow
}

\author{
A. Radhouane $\cdot$ H. Bournot $\cdot$ N. Mahjoub Said $\cdot$ \\ H. Mhiri - G. Le Palec
}

Received: 15 March 2009/Accepted: 30 August 2009/Published online: 22 September 2009

(c) The Author(s) 2009. This article is published with open access at Springerlink.com

\begin{abstract}
This paper deals with the dynamics of the flowfield resulting from the interaction of twin inclined elliptic jets with an oncoming crossflow. It will particularly focus on the impact of the initial streamwise inclination angle of the emitted jets on the different flow dynamic features. This particular side of the question is of an extremely high interest as it allows a better understanding of the mixing of the different interacting flows. A well understanding of this mechanism is likely to enable us to control the jets' trajectories, expansion, confinement, etc. all these processes are in tight relationship with the atmospheric pollution and the pollutants' dispersion which are nowadays alarming questions needing urgent constraining measures and viable solutions. The consideration of the question was first carried out experimentally by means of the particle image velocimetry (PIV) technique in order to track the evolution of the jets among the environing flow. The second step of this work consisted in the numerical simulation of the same configuration by solving the NavierStokes equations with the finite volume method and the Reynolds stress model (RSM) second-order turbulent model. The use of this particular model is in itself a new contribution in the "jets in crossflow" examination; and its merit comes from its high ability to detect the shear stresses that are precisely determinant in the mixing enhancement. The efficiency of this model was proved in our work by the
\end{abstract}

A. Radhouane $\cdot$ N. Mahjoub Said $(\bowtie) \cdot$ H. Mhiri

Unité de Thermique et Thermodynamique des procédés

Industriels, École Nationale d'Ingénieurs de Monastir,

route de Ouardanine, 5000 Monastir, Tunisie

e-mail: Nejla.Mahjoub@fsm.rnu.tn; mahjoub_nejla@yahoo.fr

H. Bournot · G. Le Palec

IUSTI, UMR 6595, 5 rue Enrico Fermi, Technopôle

de Château-Gombert, 13453 Marseille Cedex 13, France satisfying matching of the numerical results and the experimental data. Once the validation obtained, we enhanced our model by introducing a temperature gradient between the interacting flows and by injecting a non reactive fume through the jet nozzles. We also varied the initial angle of the emitted jets in order to evaluate the impact of this parameter on some dynamic characterizing features such as the global jets' plumes, the windward and the leeward jets' spread, the size, the location and the magnitude of the reverse flow region, the penetration and the deflection of the jets' trajectories, the mass entrainment of the discharged pollutants from the jets' nozzles, some of the shear stress components, etc. a thorough description of these parameters is likely to well characterize the exact progression of a given particle contained within the jets (in the case of the polluted jets) which will help us find the adequate way to control it.

$\begin{array}{ll}\text { List of symbols } \\ d & \text { Jet nozzle diameter }(\mathrm{m}) \\ D & \text { Nozzles' spacing }(\mathrm{m}) \\ f & \text { Mass fraction } \\ g & \text { Gravitational acceleration }\left(\mathrm{m} / \mathrm{s}^{2}\right) \\ G_{k} & \text { Term of production due to buoyancy forces }[\mathrm{kg} / \\ & \left.\left(\mathrm{m} \mathrm{s}^{3}\right)\right] \\ \kappa & \text { Thermal diffusivity }\left(\mathrm{m}^{2} / \mathrm{s}\right) \\ k & \text { Kinetic energy of turbulence }\left(\mathrm{m}^{2} / \mathrm{s}^{2}\right) \\ P_{k} & \text { Term of production due to the mean gradients } \\ & {\left[\mathrm{kg} /\left(\mathrm{m} \mathrm{s}^{3}\right)\right]} \\ R & \text { Injection to mainstream velocity ratio }\left(R=u_{i} /\right. \\ & \left.U_{\infty}\right) \\ S_{i j} & \text { Mean strain rate } \\ T & \text { Temperature }(\mathrm{K}) \\ U_{\infty} & \text { Crossflow velocity }(\mathrm{m} / \mathrm{s}) \\ V_{0} & \text { Injection velocity }(\mathrm{m} / \mathrm{s})\end{array}$


$\overline{u_{i}^{\prime \prime} u_{j}^{\prime \prime}} \quad$ Reynolds stress $\left(\mathrm{m}^{2} / \mathrm{s}^{2}\right)$

$u_{i}, u_{j} \quad$ Velocity components along the $i$ and $j$ directions

$u, v, w$ Velocity components along $x, y$, and $z$ directions $(\mathrm{m} / \mathrm{s})$

$x, y, z \quad$ Cartesian coordinates (m)

\section{Greek symbols}

$\rho \quad$ Density $\left(\mathrm{kg} / \mathrm{m}^{3}\right)$

$\beta \quad$ Thermal expansion coefficient $\left(\mathrm{K}^{-1}\right)$

$\varepsilon \quad$ Dissipation rate of the turbulent kinetic energy

$\mu \quad$ Kinetic viscosity $[\mathrm{kg} /(\mathrm{m} \mathrm{s})]$

$\mu_{t} \quad$ Turbulent (or eddy) viscosity $[\mathrm{kg} /(\mathrm{m} \mathrm{s})]$

$\alpha \quad$ Injection angle with reference to the free stream $\left(x\right.$-axis, $\left.{ }^{\circ}\right)$

$v \quad$ Kinematic viscosity $\left(\mathrm{m}^{2} / \mathrm{s}\right)$

$\lambda$ Thermal conductivity $[\mathrm{W} /(\mathrm{mK})]$

$C_{p} \quad$ Specific heat $[\mathrm{J} /(\mathrm{kg} \mathrm{K})]$

$\delta_{i j} \quad$ Kronecker symbol ( $=1$ if $i=j$ and 0 if $i \neq j$ )

\section{Subscripts}

$\infty$ Conditions in crossflow

$0 \quad$ Exit section of the jet

\section{Superscripts}

- Reynolds average

Favre average

\section{Dimensionless groups}

$\operatorname{Pr} \quad$ Prandtl number $\left(P r=\mu C_{p} / \lambda\right)$

Re Reynolds number $\left(R e=u_{i} d / v\right)$

Sc Schmidt number $(S c=v / \kappa)$

\section{Introduction}

Research within the realms of fluid mechanics and aerodynamics often comes across the common configuration of "jets in crossflow". Generally, it is question of single jets in crossflow due to their wide presence in several engineering applications. Nevertheless, the incessant need for increasing the efficiency of the handled applications (cooling, heating, mixing, dispersion, etc.) has multiplied the number of the emitted jets and oriented the concentration towards the "multiple jets in crossflow". The examination of the intermediate configuration; i.e. the twin jets in crossflow is, however, primordial as it shows the newly developed mechanisms (mixing between the emitted jets) and structures as well as the changes occurred on the already existing features.

The "twin jets in crossflow" configuration is frequently related to environmental concerns in applications like the smoke stacks exhausted from either industrial or vehicle (cars, ships, etc.) chimneys. It is also related to water pollution that is met in the discharge of liquid effluents through piping systems into the rivers, seas or oceans, etc. In such applications, the main goal is to be able to control the mixing and dispersion processes in order to respond to the constraints imposed by regulatory agencies. The environmental side of these applications has become so alarming that these constraints are becoming multiplied and tightened from day to day.

The well understanding and control of the mixing process is also important from a technical point of view. In fact, in applications like the injection of gas through combustion and other mixing chambers in the chemical process industries, it is highly recommended to increase the operating temperature for performance concerns. On the other side, we are always limited by the melting temperature of the handled material which acquires the cooling process a further importance.

Other uses of the jets in crossflow are evident in aerospace applications like the thrust vector control in rockets or in VSTOL aircrafts. In the latter, the need is to restore the flight comfort that is damaged by aerodynamic disturbances. They are mainly engendered by the interference of the lifting jets efflux with the surrounding mainstream during the transition stage from hover to forward flight. We see then that a better knowledge of the jets in crossflow mixing behavior is essential for the control of the emitted jets and even in the design of more efficient flow mixing systems.

Consequently to this large amount of applications making use of the "twin jets in crossflow" configuration, several authors paid attention to their different developing stages. Herein, we have to precise that the twin jets may be arranged differently towards the oncoming crossflow as they may be aligned (tandem) with the mainstream, side by side or opposite. Rare are the works that have dealt exclusively with the inline jets. To our knowledge, Ohanian and Rahai [1] are the only authors to have done this by conducting numerical investigations of two turbulent planer jets in crossflow. The main goal of their paper was the evaluation of a dynamic and a geometric parameter; the injection ratio and the jet nozzle spacing, respectively; on the resulting flow dynamics. That clearly demonstrated the absence of the jets' coupling and the enhancement of the throw distance in the crossflow before tilting at the highest jet spacing distance. The mixing is, however, enhanced under the opposite conditions; that is to say under an increase of the jets' coupling.

Disimile et al. [2] tested the influence of the same parameters; the injection ratio and the nozzle spacing; on the both single and double jet configurations. These tests were carried out experimentally by means of video digitization and managed to relate the jets' development to the imposed parameters. They also allowed the determination of the averaged jets' penetration as well as an intermittency 
function demonstrating the similarity of the handled models' behaviors.

These two configurations have also been considered by Makihata and Miyai [3] in order to compare their dynamic features. To reach this goal, experimental and theoretical predictions of the trajectories of both configurations have been carried out in the case of buoyant and non-buoyant jets in a uniform crossflow. The velocity measurements acquired under various velocity ratios and normalized distances (rate of the distance between the nozzles and the jet diameter) showed a clear transition of the outline of the velocity contour maps from circular to kidney shaped.

The work of Ibrahim and Gutmark [4] followed the same procedure since it compared the flowfield resulting from a single and a double jet in crossflow. Their results, obtained experimentally by means of the particle image velocimetry (PIV) system, were tracked under different velocity ratios. These experiments allowed the characterization of the jet trajectory and penetration, the deflection of the jet trajectory, the mass entrainment approximation based on the jet trajectory, the windward and leeward jet spread, etc. It was concluded from the observation of these features that the double jet behaves like a higher blowing ratio single jet in crossflow.

Some other authors chose to compare the double to both single and multiple jets in crossflow configurations. Ziegler and Wooler [5] are pioneers in the domain since they established this comparison since the early seventies (1973). They have even considered the question since earlier by comparing normal twin inline and side by side jets in crossflow [6]. This comparison was established by means of a physical model that was validated by confrontation with test data relative to normal and inclined $\left(60^{\circ}\right)$ twin jets in crossflow. This model could mainly explain the jets' development in terms of pressure forces and entrainment of the mainstream fluid. It also detailed the asymmetric dependence of the jets on each other since the downstream one depends closely on the upstream one whereas the contrary is not true.

The comparison of the number of the emitted jets has been conducted a little later by the same authors [5] and was based on the velocity stratification. For the matter, jets with different exit velocity stratifications have been considered: jets with a relatively high velocity core, jets with a relatively low velocity core and jets originating from a vaned nozzle. The first one was represented by a uniform exit velocity profile, the second by an ideal nozzle and the last one by a more elaborated motion equation. The confrontation of the numerical calculations to the experimental data resulted in a satisfying agreement which allowed a consistent characterization of the mutual interference effects between the jets.
A further dynamic comparison between single, double and multiple jets in crossflow behaviors has been conducted by Isaac and Jakubowski [7]. It was performed experimentally by means of hot-wire anemometry in a wind tunnel on jets characterized by a velocity ratio equal to 2 and a jet spacing of 4 nozzle diameters. The experiments proved the similarity of the downstream of the inline jets behavior with reference to the single jet one with, however, a slightly higher trajectory. This similarity was established in the far field $(x / d>10)$ in terms of mean velocity and turbulence mechanisms. These features and others like the Reynolds stress profiles also demonstrated the similarity of the single and multiple jet cases. It was finally suggested that the initial condition have significant role on the each of the jet trajectories even if they do not affect the turbulent parameters themselves.

Xiao [8] considered the same configurations with, however, the limitation of the number of the multiple jets to three. All of them are emitted within a confined crossflow with constant boundary conditions for the jets and the duct flow. In the double and triple jets system, the centre-to-centre distances between the jets were 0.68 and $0.20 \mathrm{~m}$, respectively. A blockage effect and a recirculation of the mainstream were generated and were strongly affected by the reigning parameters since they were weaker at larger jet spacing and more significant when the number of the emitted jets increased.

Direct numerical simulations (DNS) of the same single, double and triple jets in crossflow configurations have been performed by Maidi et al. [9]. The jet exit section is, however, square and the jets are emitted normally with respect to a jet to cross-flow velocity ratio of 2.5 and a Reynolds number of 225 , based on the free-stream quantities and the jet width. The DNS gave a satisfying agreement with the findings of other researchers; both numerical and experimental. The corresponding results proved the dependency of the merging process of the counter-rotating vortex pair of the twin jets on the spacing that separates them. The exploration of the triple jet configuration revealed the existence of more complicated flow interactions between individual jets and cross-flow likely to enhance their corresponding engineering applications.

At the end of this brief literature glimpse, we can say that too few works were exclusively devoted to the inline twin jets in crossflow. Most of the available papers consider rather different arrangements (side by side or opposite) and/ or different number of emitted jets (single or multiple) that are most of the time normal to the crossflow. The injection ratio and the jet spacing are the most tested parameters in order to evaluate their impact on the established flowfield and its structural features. In our work, we propose to consider two tandem jets emitted within a cooler oncoming crossflow according to a variable initial inclination angle. Our purpose is to evaluate the impact of this parameter on 
the dynamics of the resulting flowfield and more particularly on its velocity components and shear stresses. Such an examination is likely to provide us with an overall idea about the mixing process occurring between both of the jets from one side and between the jets and the environing flow on the other side. A better knowledge of the mixing process is primordial for a more efficient control of the jets' evolution (expansion, extent, elevation, etc.) which is of a high interest in all the already mentioned applications and further more in the ones that are in tight relationship with the environmental sake where the control of the toxic fumes is crucial.

The first step of our study will then consist in an experimental examination of two inline air jets within an air mainstream. A numerical simulation of the configuration is then carried out by means of the finite volume method based on the resolution of the Navier-Stokes equation by means of the RSM second-order turbulent model. The numerical results; validated by confrontation with the experimentally tracked data with the PIV technique, allowed the characterization of the resulting flowfield main dynamic features as well as its turbulent behavior that is mainly responsible for the mixing mechanism. Radhouane et al. [10] has recently considered this configuration and even evaluated the impact of the same parameter; inclination angle. Nevertheless their efforts were rather oriented towards the mass and heat transfers taking part among the interacting flows. The present work in addition to theirs is likely to give a fully developed idea about the impact of the jets' inclination on the resulting flowfield and present viable solutions to the commonly met problems.

\section{Presentation of the problem}

The emission of two tandem jets inline within the oncoming crossflow generates two different types of interactions. The first takes place between the emitted jets themselves and the second between the jets and the environing flow. Both interactions are determinant in the establishment of the resulting flowfield as they all participate in the mixing process. Structurally speaking, the mixing process manifests through the development of various vortical structures that appear at different locations of domain and can either enhance or reduce the quality of the global mixing. The initially adopted conditions and then the imposed parameters such as the jet spacing, exit section shape, the injection ratio, etc. play a determinant role on the existence, evolution and size of these structures. Nevertheless, four main features are always present regardless of these conditions, as shown in Fig. 1 [10]: the horseshoe vortices (HSV), the wake vortices (WV), the shear layer vortices (SLV) and the contrarotating vortex pair (CVP) (Fig. 1).

The horseshoe vortices (Fig. 2a) are the first to take place near the injection plate just windward of the jets to finally wrap around the jets' columns like a necklace. In an attempt to describe these vortices formation, Andreopoulos and Rodi [11] stated that when the boundary layer's main flow fluid is deflected laterally from the center plane due to the adverse pressure gradient in front of the jet, the spanwise vorticity in the boundary layer is stretched to form the front of the horseshoe vortices. They are induced by the adverse pressure gradient formed at the injection wall that forces the wall boundary layer to separate and results in this type of vortices. The latter are then stretched and convected about the periphery of the jets like a necklace: this is analogous to the vortex system resulting from the interaction of a boundary layer approaching a cylinder mounted to a wall [12]. The HSV are found to be steady, oscillating or coalescing. Frequencies of oscillation have been found to be correlated with periodic motions of upright vortices [13].

As long as the jets emerge and penetrate through the mainstream, the jets' plumes are deflected, rolled up and
Fig. 1 Progressive evolution of the twin jets during their emission through the oncoming crossflow and the associated diagram of vortex system

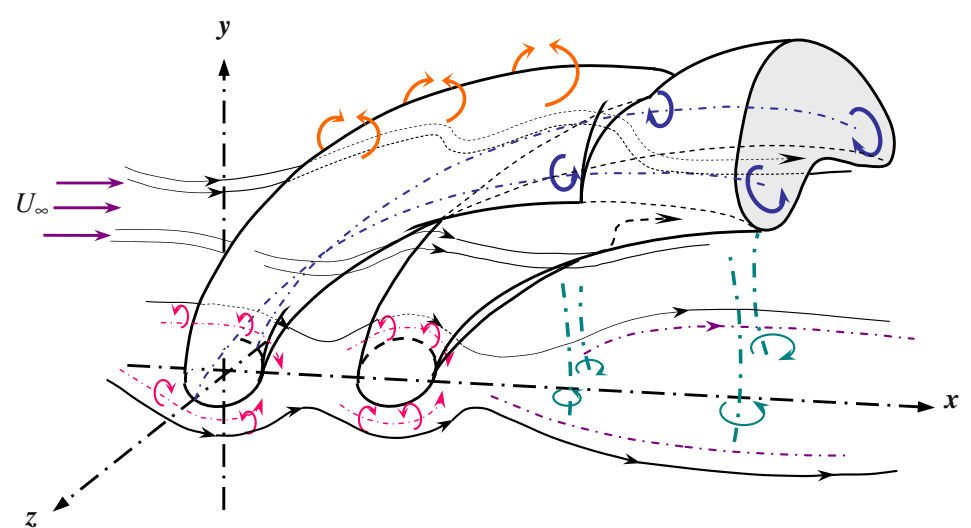

C CVP: Counter rotating Vortex Pair

C SLV: Shear Layer/ Ring like/Leading edge Vortices

C HSV: Horseshoe Vortices
C WV: Wake/Upright/Zipper/Tornado like/Fric's Vortices 
Fig. 2 Visualization of the different established vortices along the domain: a Horseshoe vortices, $\mathbf{b}$ shear layer vortices, c wake vortices, $\mathbf{d}$ counter rotating vortex pair. $\mathbf{a}$ and $\mathbf{d}$ are obtained numerically, and $\mathbf{b}$ and c are obtained experimentally for $R=1.29, \alpha=60^{\circ}$ and $D=3 d$
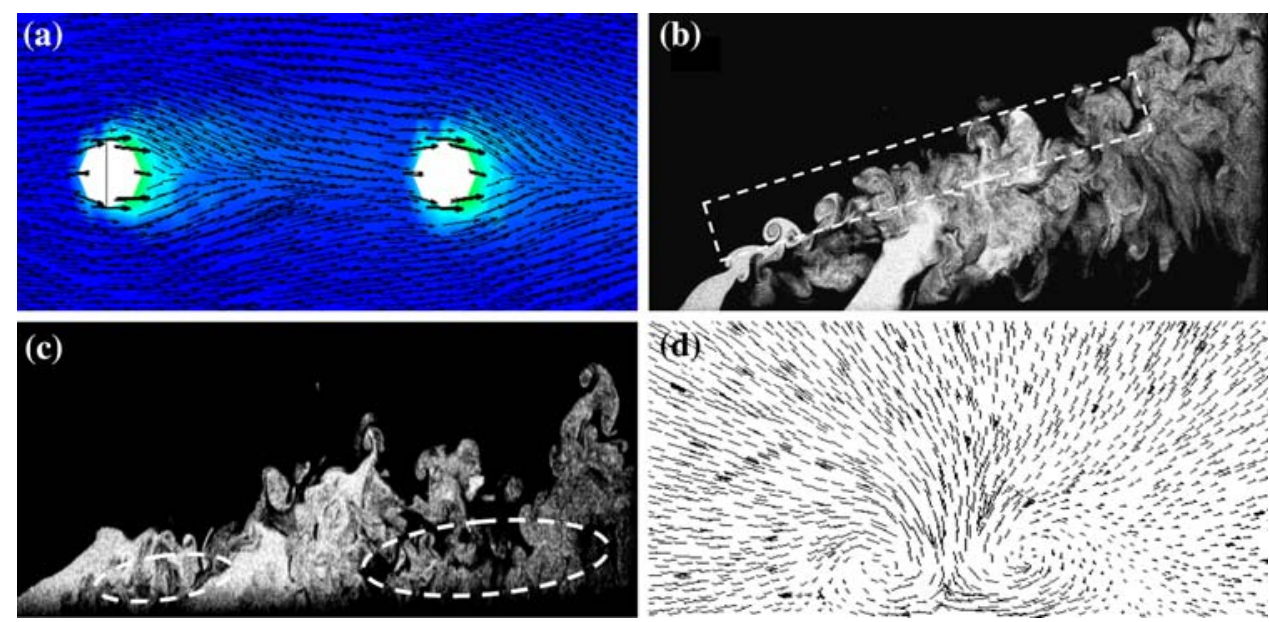

skewed resulting in the formation of jet shear layer vortices (SLV) (Fig. 2b) also known as leading edge or ring like vortices. These are quasi-steady vortices that take place in the interface between the front surface of the twin jets and the oncoming crossflow as a result of the Kelvin-Helmholtz instability of the annular shear layer that separates from the edge of the injector. Kelso et al. [14] report that the shear layer rollup is limited to the upstream side of the jet for smaller $R e$, while for crossflow Reynolds numbers higher than 1,000, large scale roll up occurs along the entire perimeter of the shear layer. These structures are analogous to the vortex ring structures typically observed in free jets. The SLV may also turn and realign itself in the streamwise direction due to the momentum of the oncoming crossflow.

Between the injected jets' lee-side and the injection plaque (wall), develop the upright vortices (UV) (Fig. 2c) also called wake vortices, zipper vortices, tornado like vortices or Fric's vortices since they were first identified by Fric and Roshko [15] by means of smoke wire visualization. The boundary layer of the crossflow has been observed to provide the main source of vorticity in the wake vortices. Fric and Roshko [15] have also identified separate events where the wall boundary layer forms vortices which attach themselves to the lee-side of the jet and eventually form the wake vortex system. This finding is called an upright wake vortex system since one end of the vortex string is connected to the jet and follows the jet trajectory whereas the other end stays close to the cross flow wall, positioning the vortex in an upright orientation. For low Reynolds numbers, they are the only present unsteady structure.

Finally when the jets' respective plumes join, a counter rotating vortex pair (CVP) appears in the mean flow (Fig. 2d); they have already appeared in the separated plumes but become more significant when they develop in the resulting plume. This vortex results from the bending of the jets in the streamwise direction and represents the most robust flow feature over all parameter ranges; that is why it has been a subject of numerous studies in the particular case of a single jet in crossflow [14, 16-18]. Though the CVP is present in the mean flow, it has significant unsteady components [19] and its instantaneous structure may be strongly asymmetric (Smith and Mungal [20]). The mechanism of the CVP's formation is not yet fully understood in a single jet in crossflow's configuration and even less in that of double jets in crossflow. Some authors have, however, tried to explain it in a two dimensional model of a single jet in crossflow like Muppidi and Mahesh [21] who stated that the deformation of the jet cross section can be explained in terms of the pressure field around the jet, and of the initial acceleration that the jet fluid experiences in the direction of the cross-flow. They also explained the experimentally observed dependence of the CVP formation on the velocity ratio with the jet acceleration dependence on the cross-flow velocity. Which is certain is that the CVP originates at the side-walls of the jets. As it is generally accepted that the jets' shear layers fold and roll up very near to the pipes exits, leading to or contributing to the formation of the CVP, although there are still remaining questions related to the nature of vortex roll up just at the nozzles' exits. It has also been stated that the tilting and the folding of the vortical structures significantly contribute to the downstream components of the vorticity which leads, on an averaged basis, to the formation of counter-rotating vortical structures. All this concern toward the origin and development of CVP is justified by its ability of controlling vorticity generation and evolution and then of controlling transverse jet mixing and, potentially, reaction processes.

An extended description of the formation and decay of these structures is available in the literature in the particular case of single jets in crossflow. The papers devoted to the twin jet structures are on the contrary very scarce [4, 22-26] and aim rather to the characterization of the 
vorticity fundamentals (turbulent vorticity flux, vorticity transport and circulation, establishment of describing formula, etc.). We propose in our paper to characterize the vorticity of the resulting flowfield by considering the shear stress distributions which are actually at the origin of the vortical structures.

\section{Experimental set-up}

The measurements were carried out in a wind tunnel (Fig. 3) using the standard PIV technique. The tunnel was $3 \mathrm{~m}$ long and its working section was $0.2 \mathrm{~m}$ width $\times 0.3 \mathrm{~m}$ height. The twin jet nozzles of diameter $d=10 \mathrm{~mm}$ were inclined and placed flush with the ground which resulted in elliptic exit cross-sections whose grand diameter is equivalent to $d / \sin \alpha$. The jets were emitted experimentally according to a streamwise angle of $60^{\circ}$ with reference to the longitudinal direction ( $x$ coordinates). The remaining experimental details concerning the geometry of the configuration, the adopted assumptions and the measurements technique are available in reference [10].

\section{Computational set-up}

The numerical side of this study is also similar to the one elaborated by Radhouane et al. [10]. We point out nevertheless that consideration was given to a steady, three-dimensional, incompressible and turbulent flow $(R e=3,333, P r=0.69$ and $S c=0.74)$. A Cartesian coordinate whose origin coincides with the center of the upstream jet system is adopted due to the asymmetry of the established flowfield in spite of the symmetry of the model geometry. The resolution of the Navier-Stokes equations (thoroughly described in [10]) needs the use of a turbulence closure model able to characterize the fluctuating

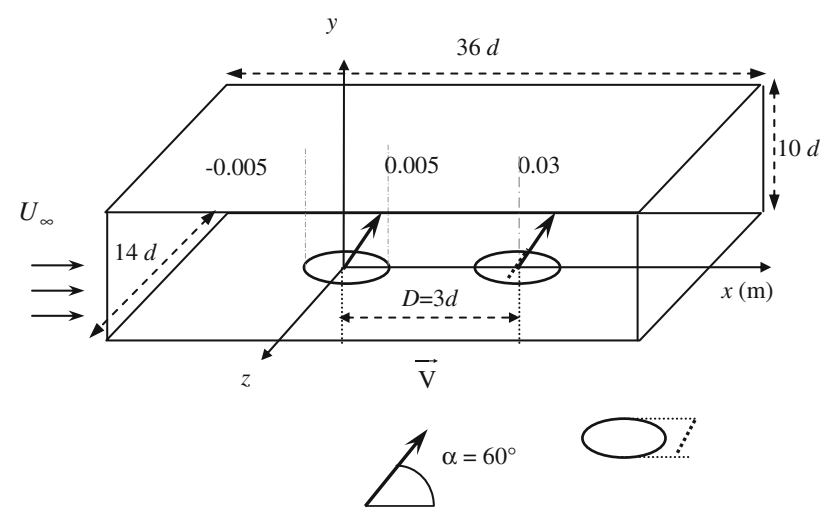

Fig. 3 Geometric disposition of the twin inclined jets among the whole domain and their relative dimensions functions. The trial of both the $k-\varepsilon$ standard turbulent model and the Reynolds stress model (RSM) second-order model together with a non uniform grid system particularly tightened in the vicinity of the jet nozzles' exit led to the adoption of the latter due to its better fitting to the experimental data [10].

The discretized equations were then solved by means of the finite volume method, the correction of the calculated pressure was carried out by means of the Patankar and Spalding's algorithm "SIMPLE" [27] and finally the convergence of the calculations was obtained when the sum of the normalized residues attained the critical value of $10^{-3}$.

Once the validation obtained [10], we generalized the adopted assumptions by introducing a non reactive fume within the jet nozzles and a temperature gradient between the interacting flows in order to approach better the real cases. The different assumed conditions are gathered in the Table 1 . The injection ratio is fixed to $R=2$, only to provide the jets with further time to evolve before tilting and combining whereas the initial inclination of the jet nozzles varies from $30^{\circ}$ to $90^{\circ}$.

\section{Results and discussion}

\subsection{Velocity field}

To begin, we propose to represent the vertical variation of the longitudinal velocity component $(\tilde{U})$ (Fig. 4) under four different initial inclination angles $\left(\alpha=30^{\circ}, 45^{\circ}, 60^{\circ}\right.$ and $90^{\circ}$ ). The profiles are plotted on the symmetry plane $(z=0)$ and in the different characterizing zones of the domain: $x=0 \mathrm{~mm}$ that is contained within the rear jet nozzles, $x=15 \mathrm{~mm}$ which is contained between the twin jet nozzles, $x=30 \mathrm{~mm}$ that corresponds to the center of the downstream jet nozzle and finally $x=50 \mathrm{~mm}$ where we are situated beyond the twin jet nozzles. These locations are independent of the imposed conditions since they are relative to the jet nozzles' locations as we have just seen. Before $x=0 \mathrm{~mm}$, there is no significant variation to consider since we are upstream of the rear jet; and then no significant process is taking place.

When we are located within the first jet nozzle (Fig. 4a), we note that the augmentation of the initial inclination affects essentially the initial value of the longitudinal velocity by reducing it progressively till it becomes null although we are inside the jet's core; the least situation happens in the case of a normal injection $(\cos \alpha=0$ when $\alpha=90^{\circ}$ ). These observations lead us to suppose that increasing the initial transverse inclination tends to straighten the jets favoring rather a vertical (toward the $y$ coordinates) expansion than a longitudinal one $(x)$. Farther 
Table 1 Boundary conditions and composition of the introduced non reacting fume

\begin{tabular}{|c|c|c|c|c|c|}
\hline Boundaries & Velocity & Temperature & $\begin{array}{l}\text { Mass } \\
\text { fraction }\end{array}$ & $\begin{array}{l}\text { Kinetic } \\
\text { energy }\end{array}$ & $\begin{array}{l}\text { Rate of } \\
\text { dissipation }\end{array}$ \\
\hline Nozzle sections & $\begin{array}{l}\tilde{u}=V_{0} \cos \alpha \\
\tilde{v}=V_{0} \sin \alpha \\
\tilde{w}=0\end{array}$ & $\tilde{T}=T_{0}=303.15 \mathrm{~K}$ & $\tilde{f}=f_{0}$ & $k=k_{0}=10^{-3} V_{0}^{2}[28]$ & $\varepsilon=k_{0}^{3 / 2} / 0.5 d[28]$ \\
\hline Crossflow & $\begin{array}{l}\tilde{u}=U_{\infty} \\
\tilde{v}=\tilde{w}=0\end{array}$ & $\tilde{T}=T_{\infty}=403.15 \mathrm{~K}$ & $\tilde{f}=0$ & $k=0$ & $\varepsilon=0$ \\
\hline Walls & $\tilde{u}=\tilde{v}=\tilde{w}=0$ & $\partial \tilde{T} / \partial y=0$ & $\partial \tilde{f} / \partial y=0$ & $\partial k / \partial y=0$ & $\partial \varepsilon / \partial y=0$ \\
\hline $\begin{array}{l}\text { Other boundaries of the domain } \\
\text { Fume composition }\end{array}$ & $\begin{array}{l}\partial \tilde{u} / \partial n=\partial \tilde{v} / \partial n=\partial \tilde{w} / \partial n=0 \\
\mathrm{~N}_{2}: 76.9 \%, \mathrm{CO}_{2}: 20.9 \%, \mathrm{O}_{2}: 1\end{array}$ & $\begin{array}{l}\partial \tilde{T} / \partial n=0 \\
.8 \%, \mathrm{SO}_{2}: 0.4 \%\end{array}$ & $\partial \tilde{f} / \partial n=0$ & $\partial k / \partial n=0$ & $\partial \varepsilon / \partial n=0$ \\
\hline
\end{tabular}

Fig. 4 Vertical distribution of the longitudinal velocity component in the symmetry plane $(z=0)$ in the different streamwise characterizing regions of the domain and under the different inclination cases

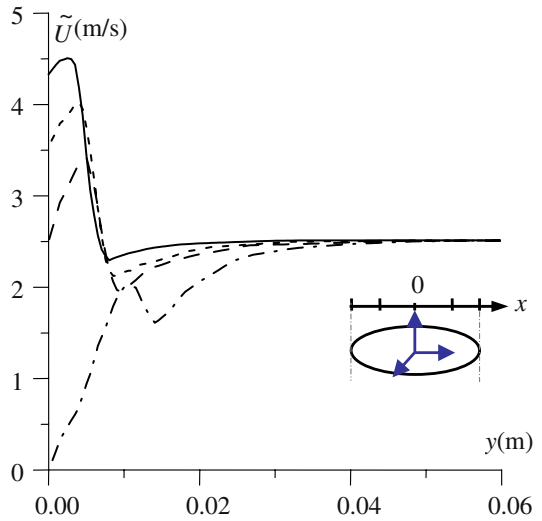

(a) $x=0 \mathrm{~mm}$

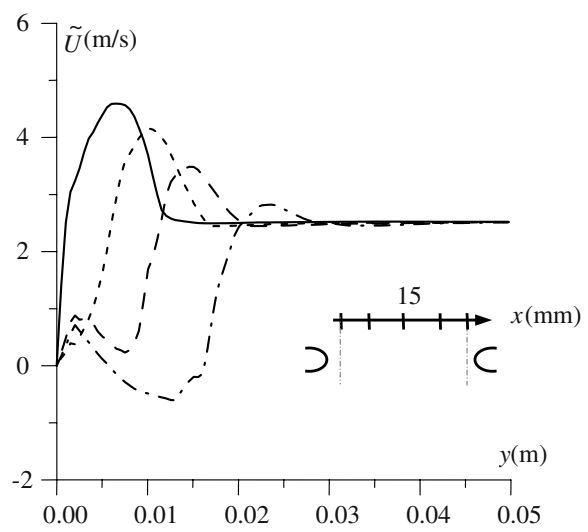

(b) $x=15 \mathrm{~mm}$

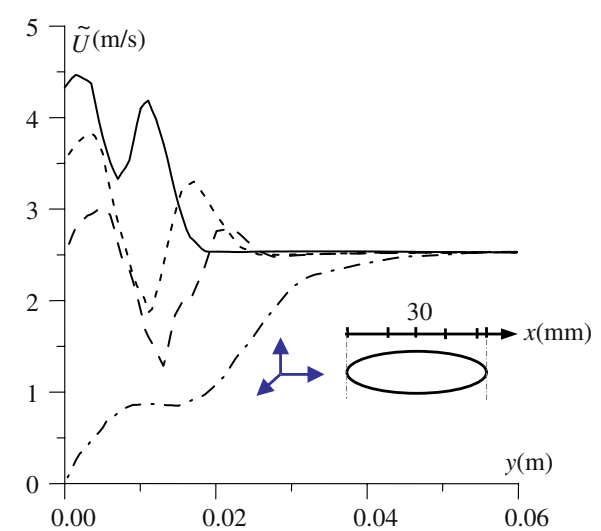

(c) $x=30 \mathrm{~mm}$

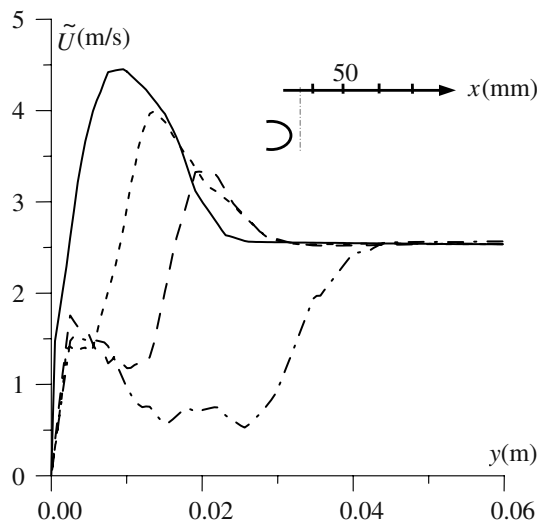

(d) $x=50 \mathrm{~mm}$ downstream, the profiles join together around a unique velocity value: the uniform velocity of the transverse flow. At that moment (in the vicinity of $y=50 \mathrm{~mm}$ ), we join the surrounding flow that stayed outside the interaction zone whose velocity is $U_{\infty}=2.5 \mathrm{~m} / \mathrm{s}$.

Between the two jet nozzles (Fig. 4b), the initial velocity is the same because in all the cases we leave the injection plaque where the velocity is null and as we progress deeper in the domain (increasing y), the behavior varies with the injection angle. In fact, for the weakest one, for example, the jets are immediately tilted by the oncoming transverse flow; leading to an immediate increase of the velocity component. When the jets are straightened, their respective plumes go deeper vertically 
first before being tilted; leading to the delay of the velocity increase. We note as well the reduction of the registered velocity peaks since we reach them after a certain distance; meanwhile, the jet's plume has lost some of its scale. Before reaching the peak in question, there is a deceleration in the velocity evolution that becomes wider (on the $y$-axis) and deeper (weaker velocity values). This is due to the same reason mentioned above, in fact, the rising of the inclination angle makes the plume of the jets flee further the injection plate; and so it is the case for their respective wake regions. So when we quit the injection plate, the velocity is first rising; but when we cross the wake region of the rear jet, a decline is noted on the velocity progression; this decline is intensified with the augmentation of the inclination since this augmentation engenders a wider wake region.

When we move to the third region; that is to say the downstream jet (Fig. 4c); we detect the presence of two velocity peaks relative to the passage through the twin jets' plumes. The peaks are more distinct, closer one-to-another and maximum for the weakest injection case. In fact, under this condition, both jets are kept near the injection plate; and so is the case for their velocity magnitude; in other words, the jets do not expand too far from the plate that is why we detect their strength almost unchanged. Whereas when the jets are straightened, they expand deeper in the $y$ direction before being tilted; which leads to the weakening of the registered peaks and their removing away. In the highest injection case, we no longer observe a velocity decline; as herein we are almost all the time crossing the jets' cores and as soon as we quit the one relative to the rear jet, we join that of the downstream one, to finally join the surrounding flow whose velocity is the highest $\left(U_{\infty}\right)$.

When we are located downstream of the twin jets (Fig. 4d), the longitudinal velocity component behaves approximately the same as between the jets' nozzles. Nevertheless we note some discrepancies, like the absence of the negative values we found between the jets. We also detect a slower homogenization of the flow since the uniform velocity is reached later $(y=45 \mathrm{~mm}$ rather than $y=28 \mathrm{~mm}$ ).

We are going to proceed the same with the vertical velocity component (Fig. 5) by considering its vertical evolution on the symmetry plane $(z=0)$. At first sight, we note that the velocity behavior within the twin jets' nozzles is globally analogous since we are always starting with a maximum value corresponding to that adopted initially to finally drop and vanish when the flow becomes homogenized. The only discrepancy consists in wider profiles and then a slower vanishing of the velocity; the total vanishing of the velocity happening at approximately $y=100 \mathrm{~mm}$ in the downstream nozzle rather than $y=80 \mathrm{~mm}$ in the rear one. It comes from this that the inclination impact on the vertical velocity appears through the initial value adopted by this feature and then by the slower homogenization of the velocity flow; even if the slope of the decrease remains the same.

Between the jet nozzles, the inclination impact is clearer and more significant since we see that under the two-first inclination angles, the velocity attains a single peak. The latter corresponds to the crossing of the extended plume of the rear jet. The rising of the reached value is due to the higher impulse brought to the jet by the initial inclination since the velocity ratio is maintained constant. Straightening more the jets brings a second velocity peak that originates from the backward expanding of the downstream jet. The amplitude of the newly added maximum is different from the already existent one but not too far from it. In this particular location, the inclination factor seems also to widen the velocity profiles which results in a slower homogenization of the velocity of the resulting flowfield.

When we go beyond of the twin jet nozzles, the behavior of the vertical velocity remains unchanged under the twofirst inclination cases. The change occurs in the following cases where a second peak develops along the velocity profile; the first one being much higher under $\alpha=60^{\circ}$ while the contrary happens under the highest inclination angle $\left(\alpha=90^{\circ}\right)$. This may be explained by referring to Fig. 8. In fact, when $\alpha=60^{\circ}$ we assist to the backward expanding of the second jet. Due to the presence of the rear jet, this backward expanding is actually transformed into a backward-lateral expanding as shown by the dashed lines on Fig. 8c and d. Since we are still too close to the injection plate this reverse flow is still not yet fully developed but if we flee further in the direction of increasing $y$ coordinates we will certainly notice it more clearly. When the inclination angle attains $90^{\circ}$ (the maximum tested angle), the jets are brought an impulse that guides them rather vertically than longitudinally, resulting in the least expanding in the $x$-direction and on the contrary in the most important backward expanding: that gives rise to a much higher second peak. Furthermore, we see that the homogenization is once more delayed since it occurs in the vicinity of $y=80 \mathrm{~mm}$ in stead of $y=60 \mathrm{~mm}$ when we are located within the jets' nozzles.

The influence of the inclination factor on the vertical lifting of the jets is explored in Figs. 6 and 7. The first one represents the lateral distribution of the vertical velocity component within the twin jets' locations at different vertical levels: $y=2 \mathrm{~mm}, y=5 \mathrm{~mm}$ and finally $y=8 \mathrm{~mm}$ (Fig. 6). The first plane provides the immediate behavior of the jets after their emission, the second follows the next evolution step and the final one tracks the last stage before vanishing, in other words before the total homogenization of the resulting flowfield. We did not flee the injection plate much further because beyond of $y=8 \mathrm{~mm}$ most variations 
Fig. 5 Vertical variation of the vertical velocity component $\tilde{V}$ on the symmetry plane $(z=0)$, in the different longitudinal locations $(x)$ and under the different initial inclination angles

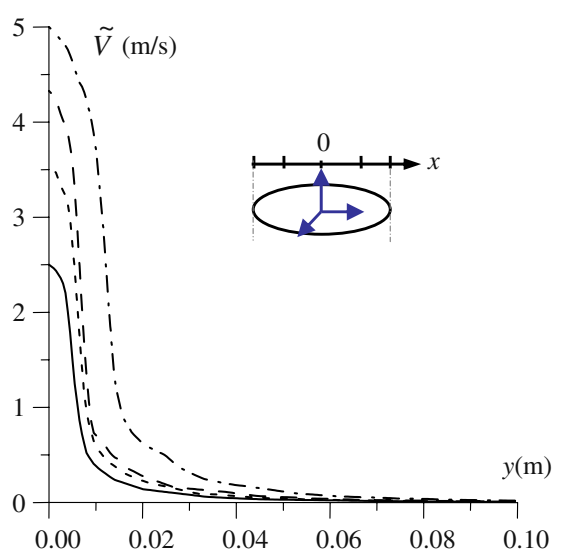

(a) $x=0 \mathrm{~mm}$

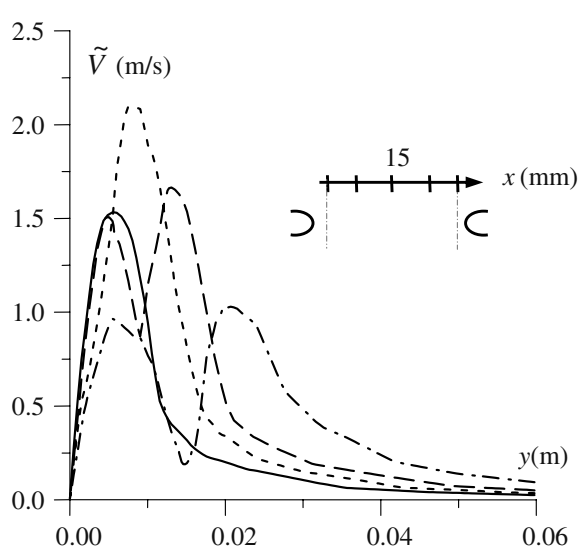

(b) $x=15 \mathrm{~mm}$

$\longrightarrow \alpha=30^{\circ}$

$---\alpha=45^{\circ}$

$-\alpha=60^{\circ}$

- - $\alpha=90^{\circ}$

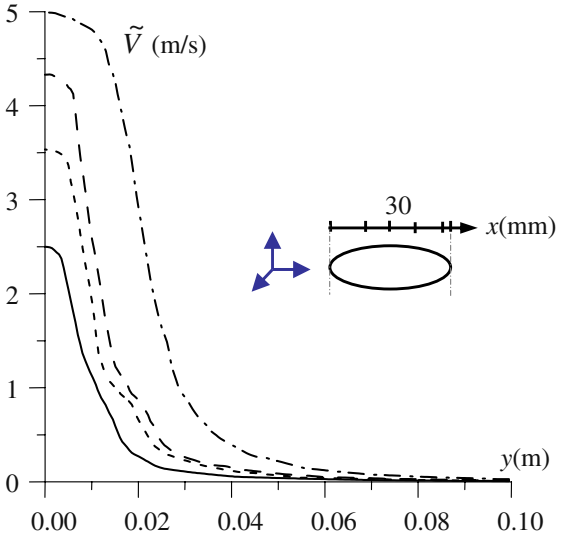

(c) $x=30 \mathrm{~mm}$

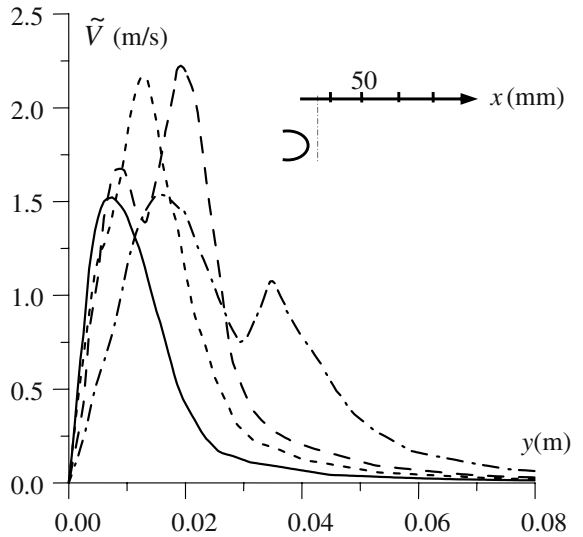

(d) $x=50 \mathrm{~mm}$ completely disappear resulting in unchanged profiles: the initial inclination could no longer allow the jets to expand higher and reach that region; thus we are out of any interaction zone. This examination will be divided into two steps: the first will concern what happens within the twin jets' locations and the following one what occurs elsewhere.

A global sight to the plotted distributions of the vertical velocity component within the primer locations shows Gaussian profiles that attain higher peaks as the initial inclination angle grows. Nevertheless this growing differs with the considered $y$-plane and the longitudinal location. In fact, when we are within the upstream jet and on the closest plane to the injection nozzles, the velocity peak sustains during a whole stage giving to the profile a square trend. The distance over which the peak is maintained constant corresponds to the injection hole dimension ( $d=10 \mathrm{~mm}$ ). The impact of the inclination angle at this level appears through the increase of the registered peak as the jets are straightened. Out of this zone and before completely vanishing the velocity accuses a decline that is more important in magnitude (deeper) and wider when the inclination angle increases. That is simply due to the fact that under a weak inclination angle, the first jet is rapidly tilted and flattened by the oncoming crossflow; leading to a rapid vanishing of the velocity. Whereas when the jets are straightened, they have a stronger impulse to flee the injection plate. That results in a higher velocity gradient leading to a wider reverse flow region and weaker reached values and then to deeper declines for the highest inclinations. These observations are comforted on the higher vertical planes since we note much deeper declines on both sides of the jet when the injection initial inclination is increased. On the other hand, we note the transformation of the square staged profiles into Gaussian ones attaining progressively decreasing and spaced maxima. The global decrease of all the profiles is engendered by the flattening of the jets under the influence of the oncoming crossing 
Fig. 6 Impact of the streamwise inclination on the lateral evolution of the vertical velocity component within the twin jets' nozzles

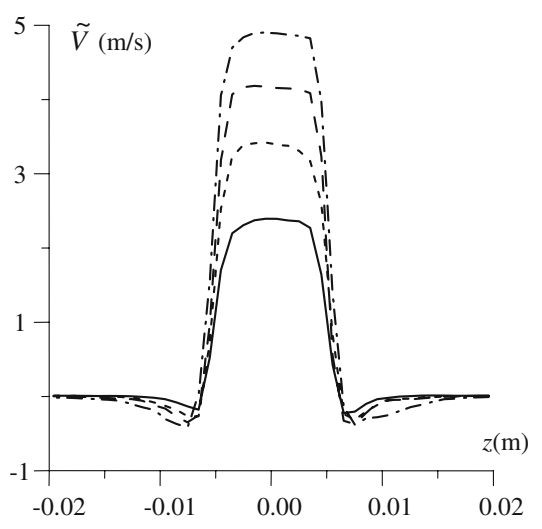

(a) $y=2 \mathrm{~mm}$
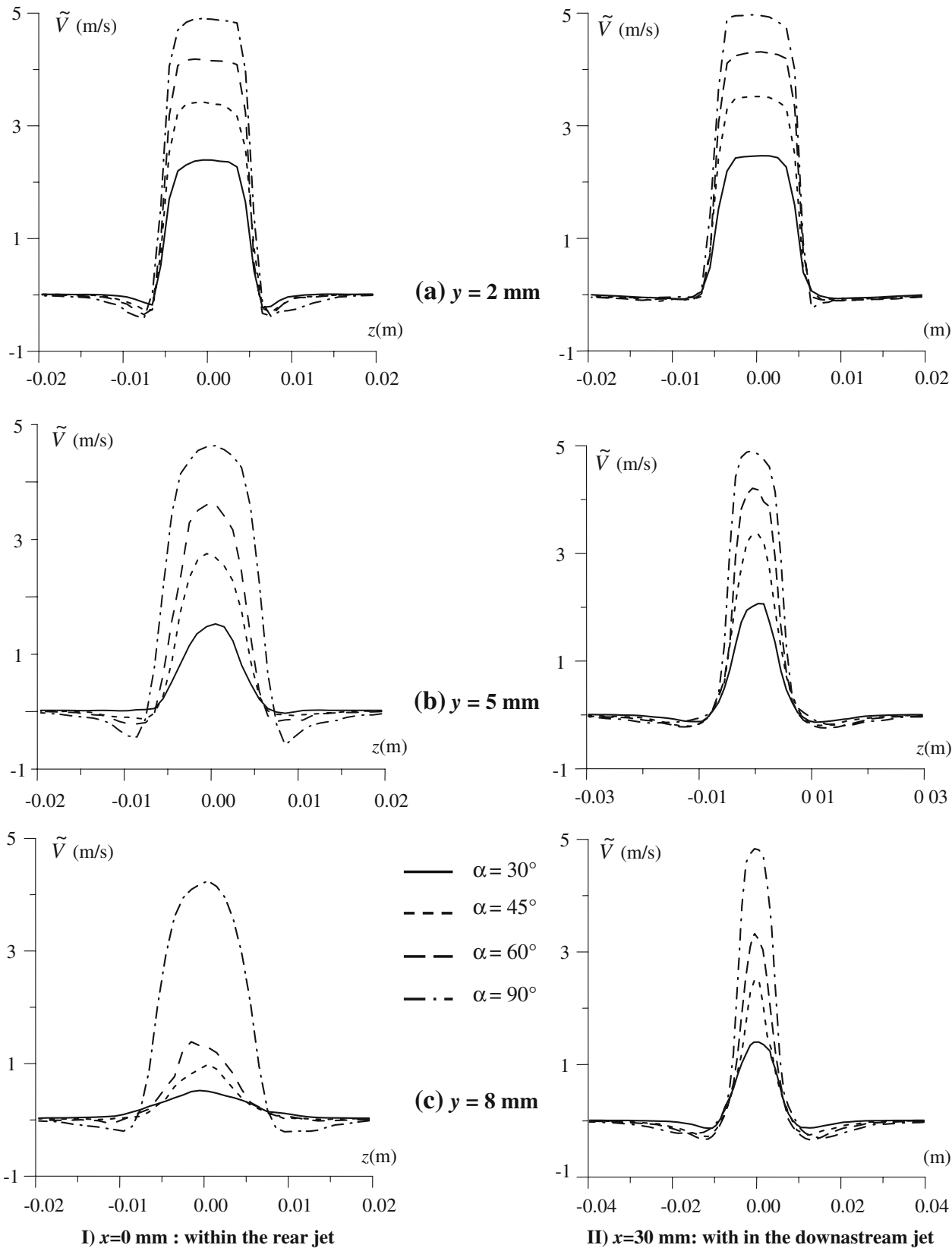

(b) $y=5 \mathrm{~mm}$
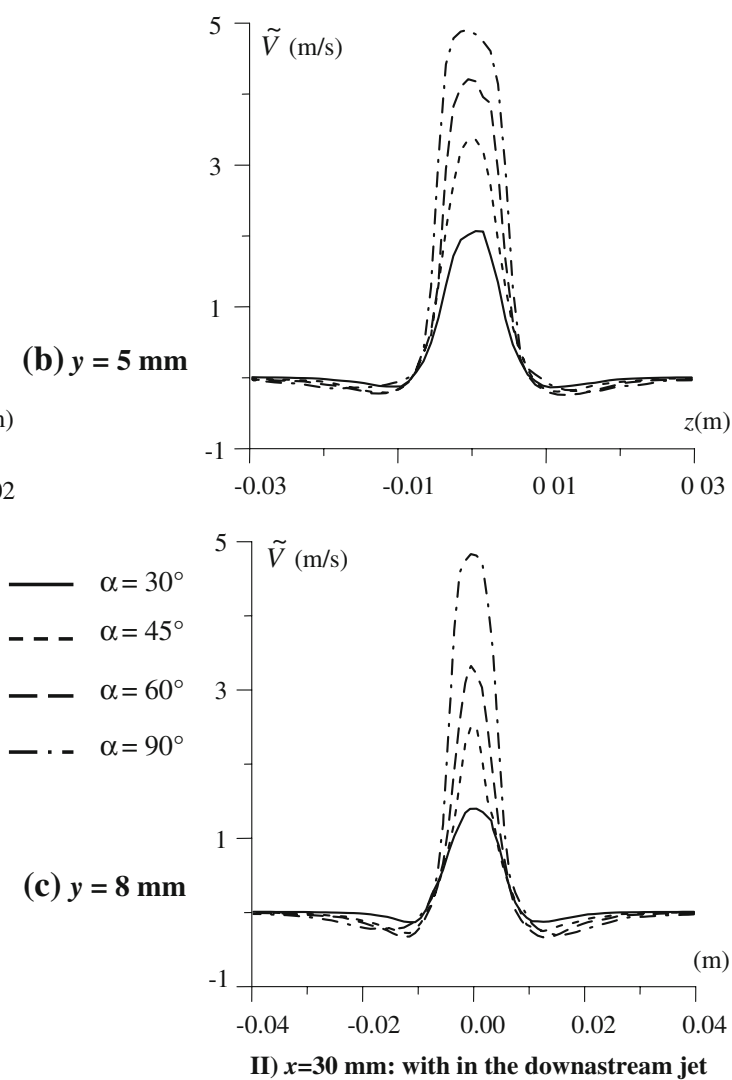

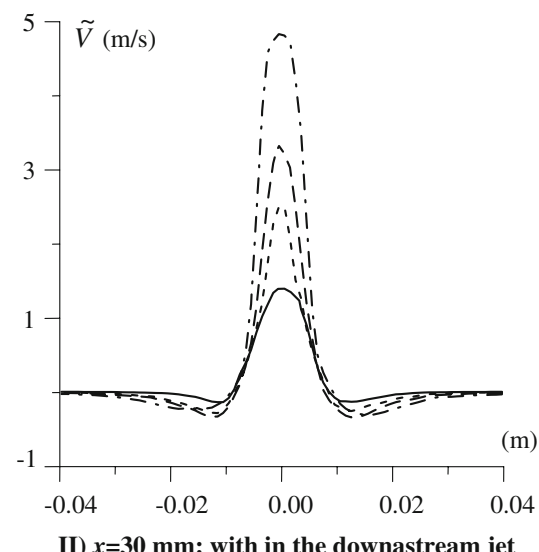

II) $x=30 \mathrm{~mm}$ : with in the downastream jet flow. However, this increase is more pronounced under the weakest inclination angle since the latter provides the jet with the least impulsion to cross the environing flow. Thus when we flee further the injection plate the detection of the jet velocity becomes harder to practically vanish on the highest plane $(y=8 \mathrm{~mm})$. On this particular plane, we see that the velocity profile of only the highest injection angle still maintains its "strength" but if we move much higher (increasing $y$ ) we will certainly assist to the total vanishing of the velocity under this angle too: that would mean that we have reached a region where only exists the crossflow that stayed out of the interaction zone.
If we move to the next jet, the same dynamic behavior is maintained with, however, some minor discrepancies. First, whereas the intensity of the attained peaks remains unchanged on the closest plane $(y=2 \mathrm{~mm})$, it accuses some augmentation on the following ones. This is due to the shielding effect provided by the rear jet on the downstream one, allowing it to maintain its "strength" much higher and farther. This same reason results in a less flattening of the second jet and then in the lessening $(y=8 \mathrm{~mm})$ or even in the absence $(y=5 \mathrm{~mm})$ of the negative decline occurring on both sides of the jet's borders. Finally, we detect the general thinning of the velocity 
Fig. 7 Impact of the streamwise inclination on the lateral evolution of the vertical velocity component between and downstream of the twin jets' nozzles

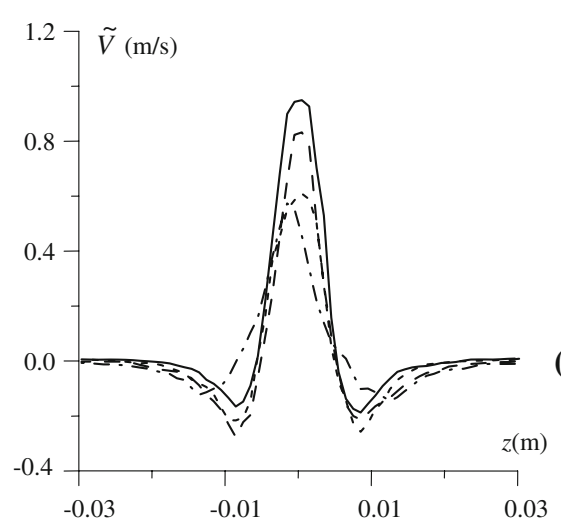

(a) $y=2 \mathrm{~mm}$
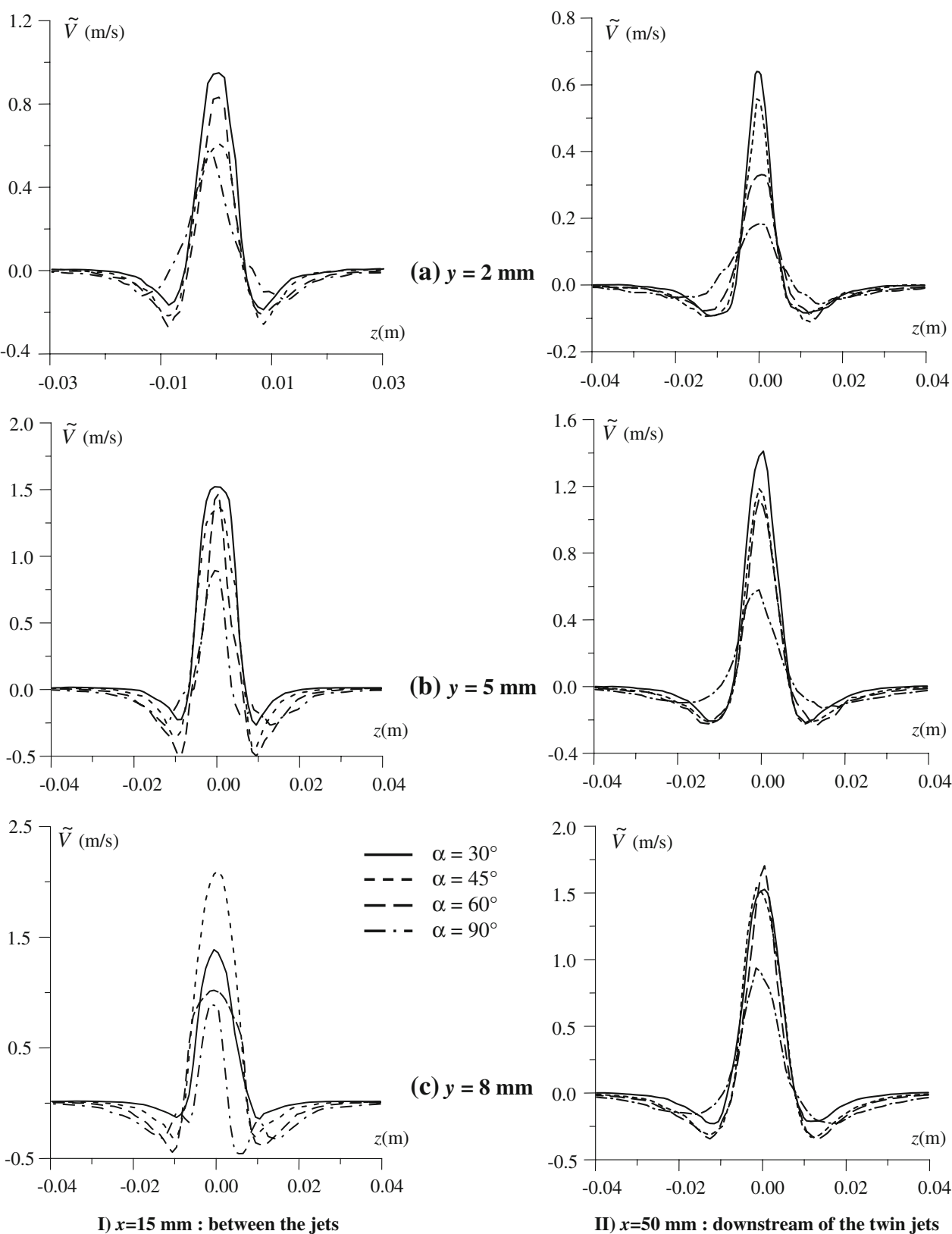

(b) $y=5 \mathrm{~mm}$
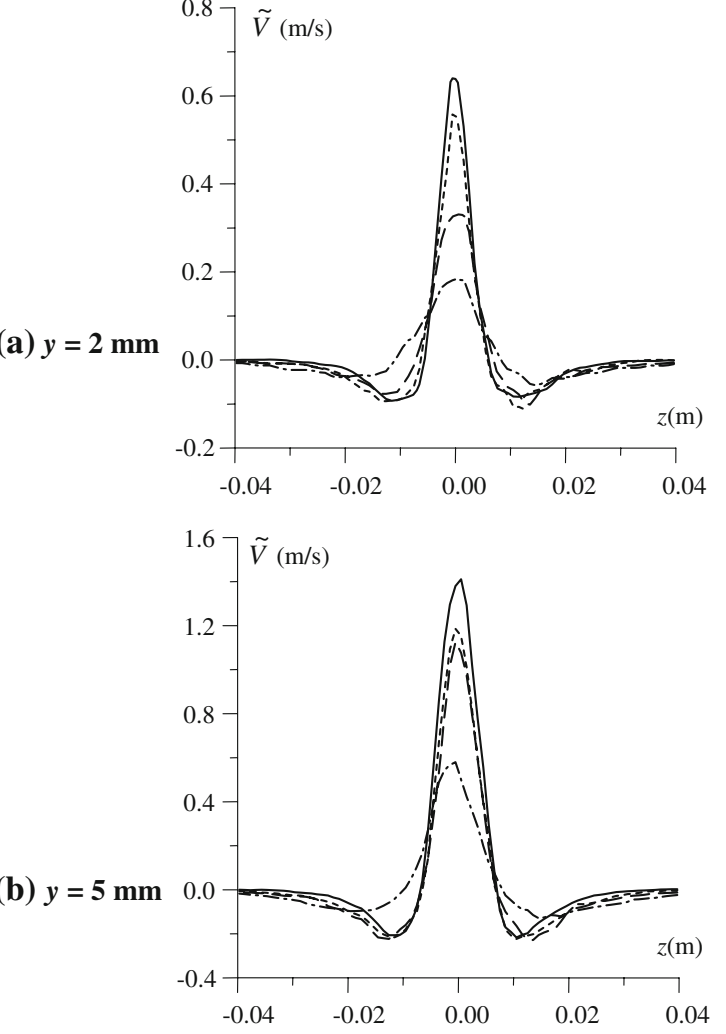

$\alpha=30^{\circ}$

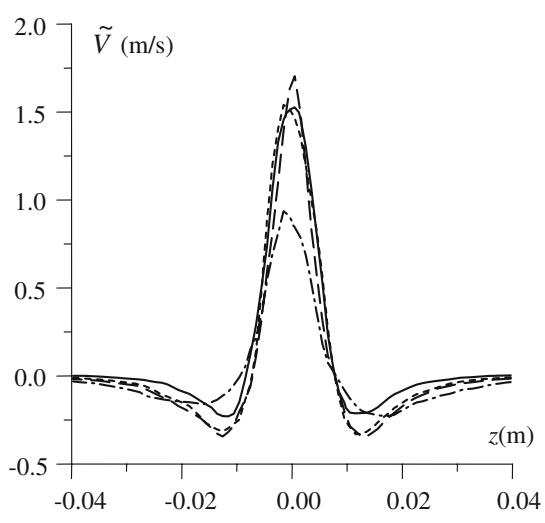

II) $x=50 \mathrm{~mm}$ : downstream of the twin jets profiles always because of the flattening reduction of the downstream jet.

The first discrepancy that appears between the profiles plotted within the jets' locations and elsewhere (Fig. 7) is the order of their superposition. In fact, whereas the velocity dominates under the highest inclination angle within the jets' nozzles; it is rather the opposite that takes place between and beyond them. Let us examine more closely the evolution within these two locations. In the first one for example and immediately downstream of the nozzle's exit ( $y=2 \mathrm{~mm}$ ), we see that the vertical velocity component responds to a Gaussian profile whose peak is decreasing as the jets are straightened. This decrease results from the combined effect of the oncoming crossflow and the first impulse brought by the initial inclination. In fact, under the weakest inclination case for example, the rear jet does not have a strong impulse that allows it to cross the environing flow, that is why it is almost immediately flattened and reoriented by the crossflow. This reorientation concerns all the jet's features and the velocity magnitude is precisely the handled one in this case; that is why all the potential core of the velocity is tilted in the crossflow's direction before being dispersed and that is finally why we don't detect its "strength" within the jet's location, but rather later when we are located within the twin jets' nozzles or beyond them. As the jets are straightened, they are brought a stronger initial impulse to cross the transverse flow (increasing $y$ ) and then loose much of their velocity 
magnitude before reaching the $x=15 \mathrm{~mm}$ location leading to the decline of the registered peak.

On the other hand, we find that the decline of the velocity on both sides of the symmetry plane is not regular and does not follow any determined order like within the jets' nozzles and that on the different examined vertical planes. On the first one for example $(y=2 \mathrm{~mm})$, even if the decline slope is approximately maintained the same, the velocity reaches decreasing negative values under the three-first inclination angle to finally increase under the highest one. These negative values are due to the presence of a horseshoe vortex that interlaces the jets' columns as shown in Fig. 8 where we plotted the velocity contours only on the closest plane and for the different angles. These vortices are said [11] to originate from the pressure gradient between the jets and the environing flow pressures. Since the mainstream is emitted according to a constant uniform velocity, varying the initial jets' inclination results in a varying jet velocity $\left(u_{i}=V_{0} \cos \alpha\right.$ and $\left.u_{j}=V_{0} \sin \alpha\right)$ and consequently in a varying pressure gradient. Nevertheless, even if the latter is much more increased under the highest inclination case, that does not generate a deeper decrease of the velocity but on the contrary a shallower one: that may be due to the higher evolution of the jets under this case and then the generation of a thinner horseshoe vortex on the considered plane. As a consequence, a weaker development of the velocity takes place on both sides of the symmetry plane $(z=0)$. The progression towards the following vertical plane $(y=5 \mathrm{~mm})$ does not bring too much changes on the velocity profiles; except the accentuation of the decline on both sides of the symmetry plane, a global increase of all the registered peaks and their further closing. This is still possible since the rear jet's impact is still "strong" to be detected on this plane. The augmentation of all the registered peaks is due to the accentuation of the velocity gradient when we reach the $x=15 \mathrm{~mm}$ location; as mentioned above that occurs due to the tilting of the whole velocity magnitude under the crossflow's impact towards increasing $x$ locations. The accentuation of the decline on both sides of the symmetry plane is also engendered by this process since a higher velocity gradient generates a higher pressure gradient, then a wider reverse flow region and finally less velocity values. On the highest plane and always within the jets' location, all the registered peaks keep on increasing apart from the one relative to the weakest inclination angle: this is only due to the fact that the jet is no longer able to flee much more the injection plate: its initial impulse brought by the initial inclination is no longer sufficient to do it. As it was the case between the twin jets' locations, moving beyond of the twin jets does not bring any significant changes.

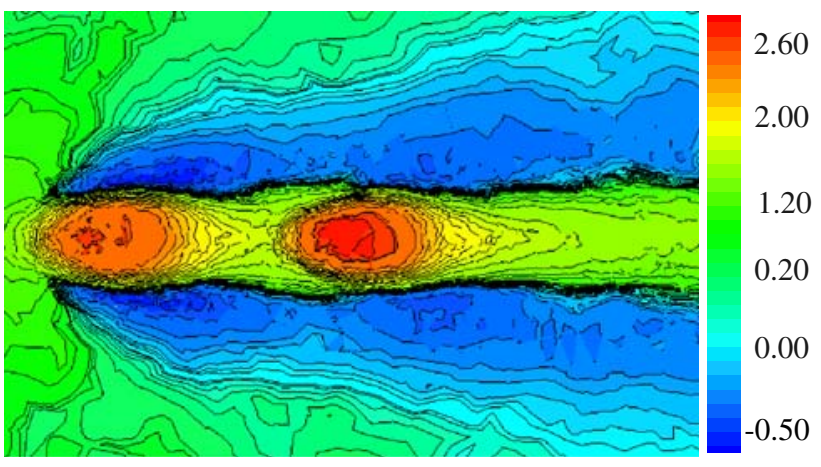

$\alpha=30^{\circ}$

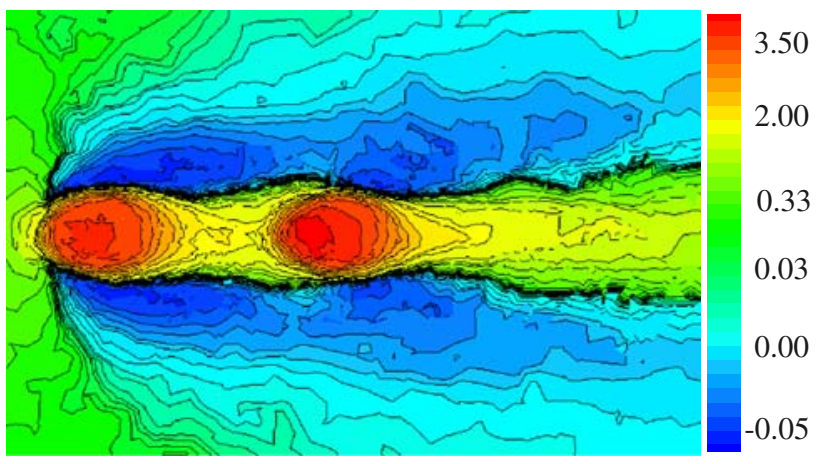

$\alpha=4^{\circ}$

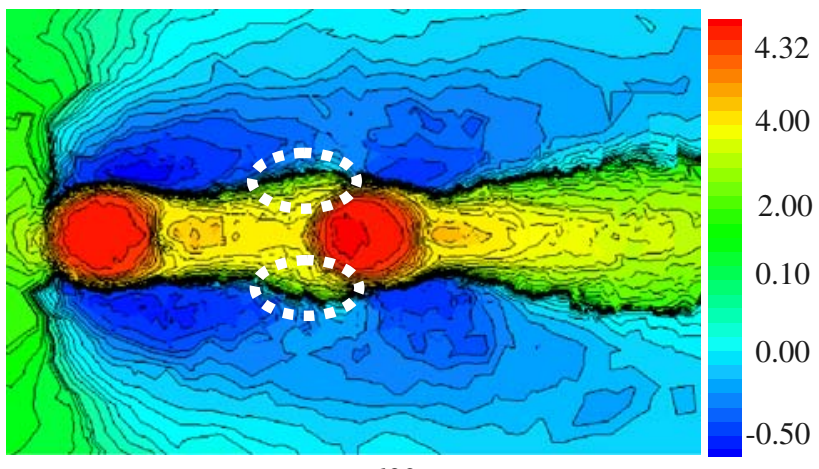

$\alpha=60^{\circ}$

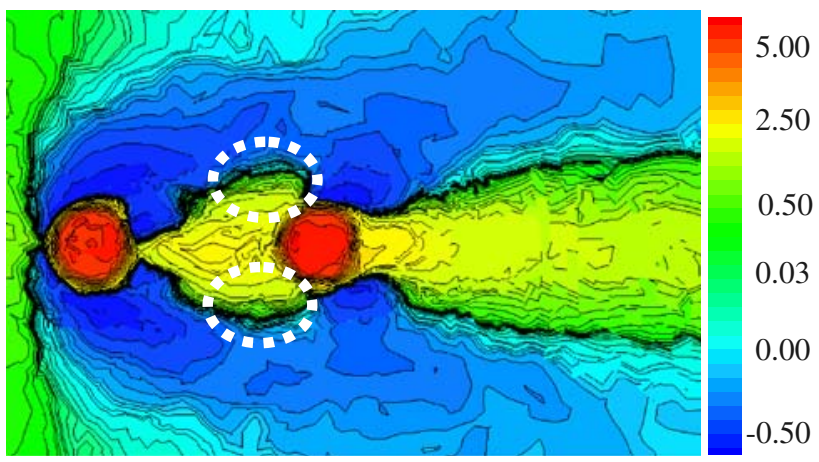

$\alpha=90^{\circ}$

Fig. 8 Visualization of the vertical velocity contours on the vertical plane $y=2 \mathrm{~mm}$ under the different inclination cases 
Fig. 9 Impact of the streamwise inclination on the lateral evolution of the spanwise velocity component within the twin jets' nozzles

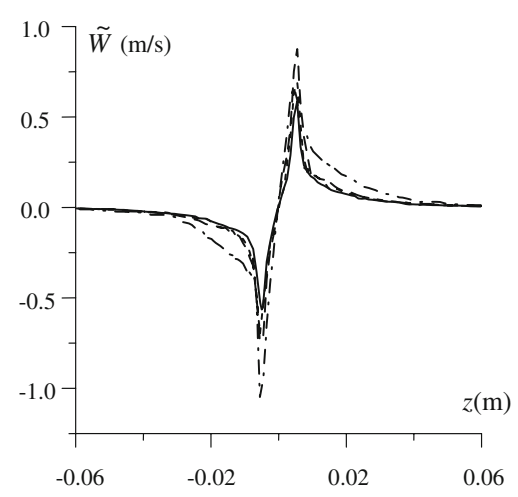

(a) $y=2 \mathrm{~mm}$
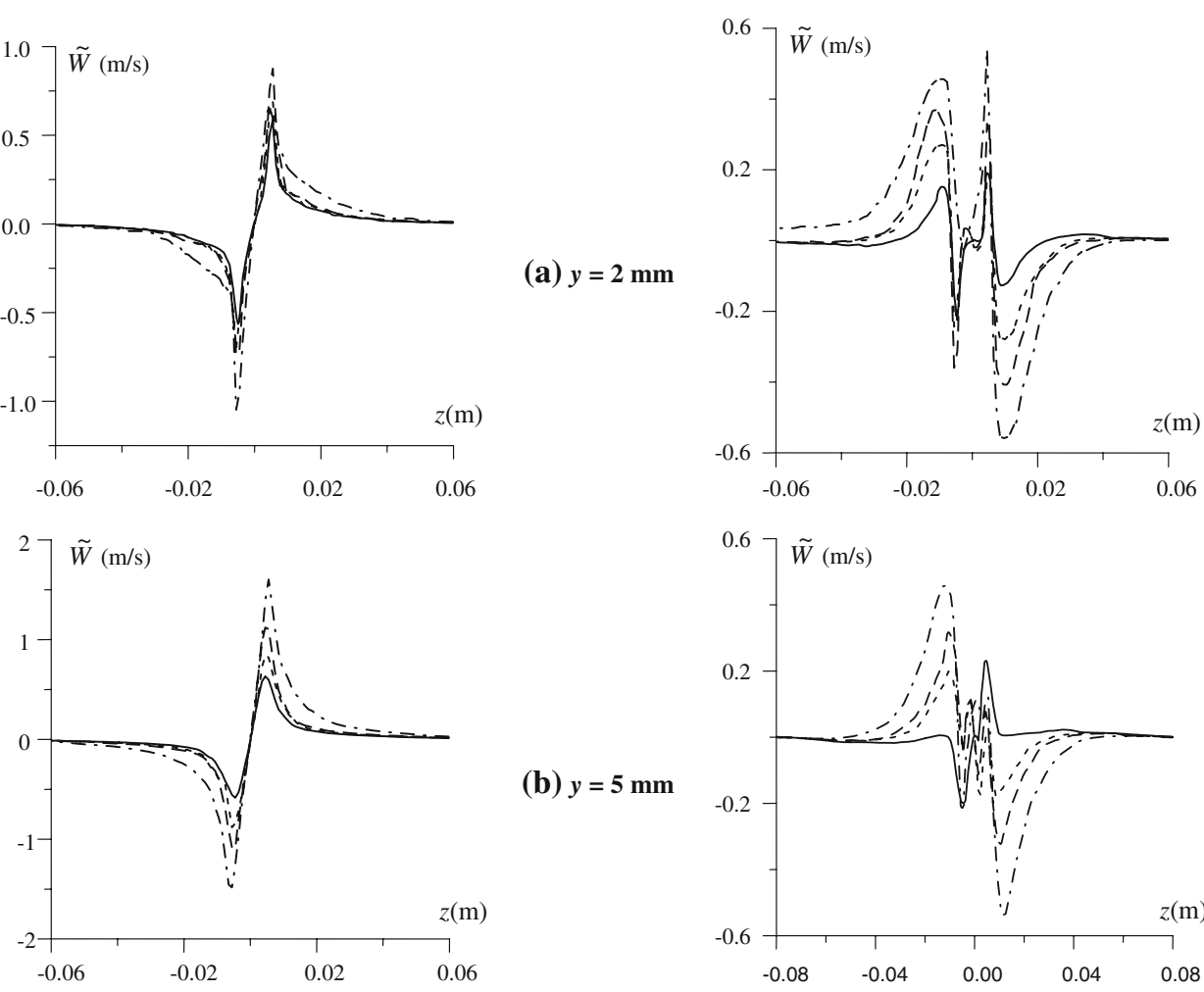

(b) $y=5 \mathrm{~mm}$
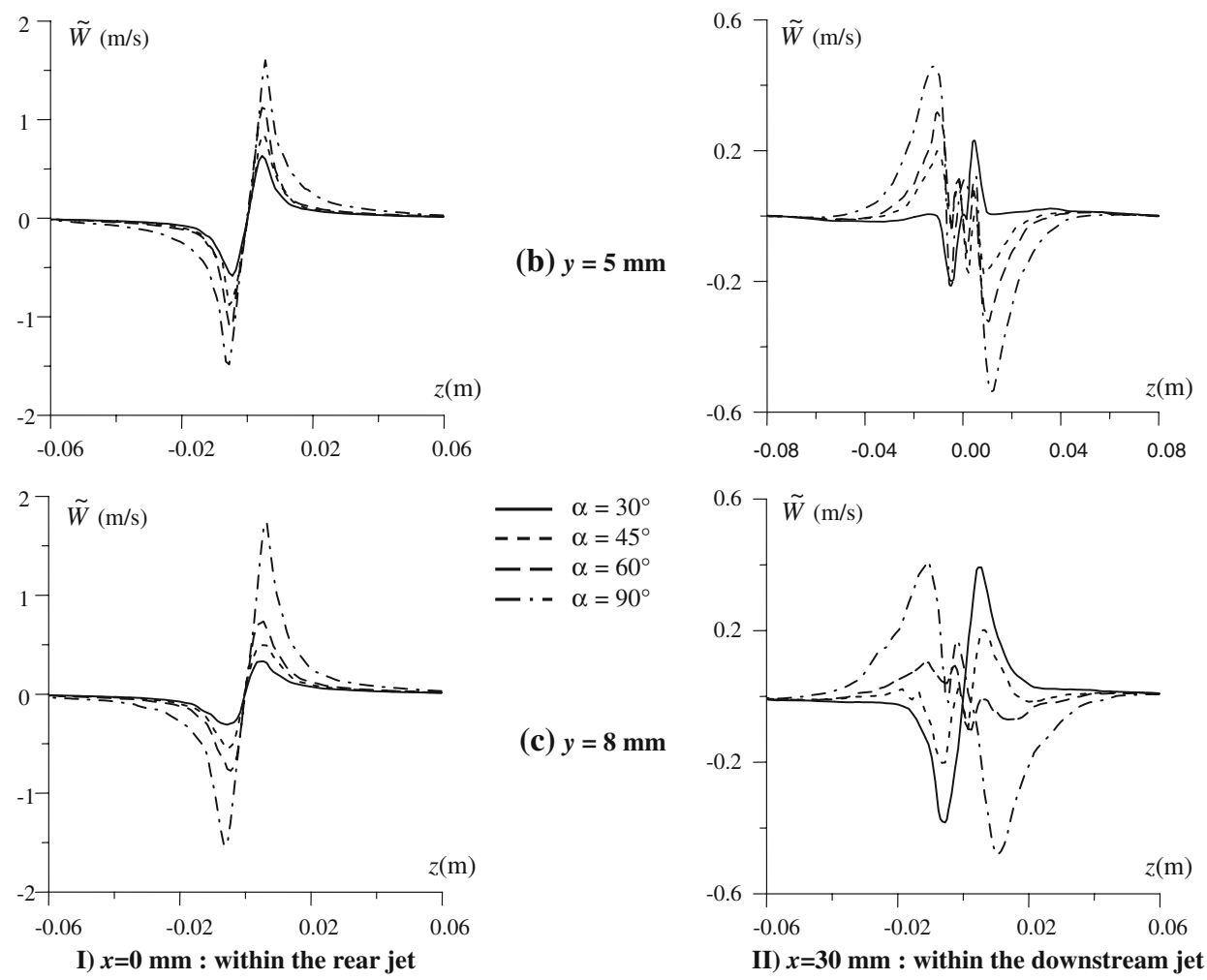

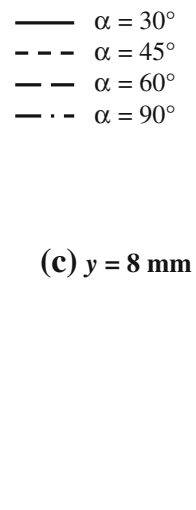

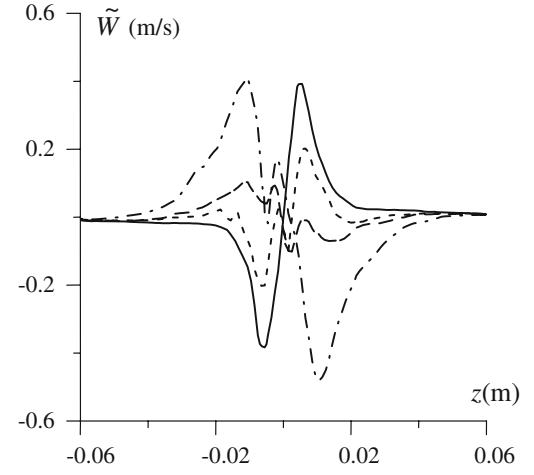

II) $x=30 \mathrm{~mm}$ : within the downstream jet
In fact they are similar to the ones brought when moved from the rear to the downstream jet and consist in the thinning of the variation profiles and the accentuation of the decline on both sides of the symmetry plane. The registered peaks, however, accuse a decline contrary to what happens within the second jet's location. Even if we cross the combination of the twin jets' plumes, we no longer attain sufficiently high peaks. This is due to the farther location we are located in $(20 \mathrm{~mm}$ downstream of the second jet rather than $15 \mathrm{~mm}$ downstream of the upstream one) and then to the vanishing of the velocity when it reaches this position. The second important feature to note here is the closing of the profiles that leads the inclination to practically no longer have any significant effect. In fact in this position and particularly on the highest plane the jets do no longer have enough "impulse" to reach it.
Thus the straightening of the jets between the jets tends to increase the registered peaks, to widen the profiles and to accentuate the decline on both sides of the symmetry plane when we are located within the jets' location. Between and beyond them, it is rather the contrary that takes place with, however, maintaining the accentuation of the decline on both sides of the symmetry plane.

To visualize well the three dimensionality of the problem, we are going to consider the behavior of the lateral velocity component on the transverse direction (z). As for the vertical velocity component we are going to follow this feature on different vertical levels within the jets' locations and elsewhere. We note on Fig. 9 (within the jet nozzles) that this feature is particularly complex since it adopts a proper attitude in each region of the domain. Its development is even different from one jet location to another 
contrary to the already examined velocity components $(\tilde{U}$ and $\tilde{V}$.) Within the first jet location for example, we see that the lateral velocity component attains two opposite peaks on both sides of the symmetry plane $(z=0)$. The presence of these peaks is comforted on Fig. 10 where were plotted the lateral velocity contours under the different tested initial injection angles on a plane close to the injection nozzles (situated at $y=2 \mathrm{~mm}$ ). The variation of the initial inclination seems to have no significant impact on the lateral velocity in this location since no valuable variation is present; a slight variation is, however, noticed under the highest inclination angle and consists in higher attained peaks and a slower decay of the velocity on both sides of the jet nozzle. That shows that straightening the jets leads, even if located within the jet, to a higher penetration of the jet into the crossflow and then to an enhanced mixing which delays the reaching of the flow remained out of interaction with the emitted jet. When we flee the injection plate (increasing $y$ ), the jet has enough time to evolve and be deflected under the influence of the oncoming crossflow. At this stage the initial inclination adopts a more pronounced role since under the weakest angle the jet is rapidly flattened and then attains low peaks; whereas when the jets are straightened, they can cross the environing flow deeper before loosing their magnitude. Fleeing more the nozzles' exit ( $y=8 \mathrm{~mm}$ ), does not bring changes on the highest angle case since the attained peaks remain at the same level. The changes occur on the remaining cases since a weak initial inclination does not allow an unlimited lifting of the jet. As a consequence the initial "impulse" brought by the inclination factor progressively declines and its decay rate is of course more pronounced under the weakest case $\left(\alpha=30^{\circ}\right)$.

Being located within the second jet nozzle brings a significant change on the distribution of the lateral velocity component. It consists in the development of two further peaks of opposite signs and located on both sides of the already present ones. These supplementary peaks clearly express the presence of the double jet since the interior peaks correspond to the second jet emission whereas those located on the periphery correspond to the extension of the reverse flow trapped between the twin jets' columns (Fig. 10). The passage to a higher plane $(y=5 \mathrm{~mm}$ ), brings changes on the development of the internal and external peaks. In fact, whereas the highest internal peaks are reached with the highest inclination angle on the first plane, this order is completely inversed in the current plane since the highest peaks correspond to the weakest angle. Furthermore, we note the disappearing of the periphery peaks under the weakest inclination case.

This disappearing is more pronounced on the highest plane where it fully concerns the two weakest angles cases
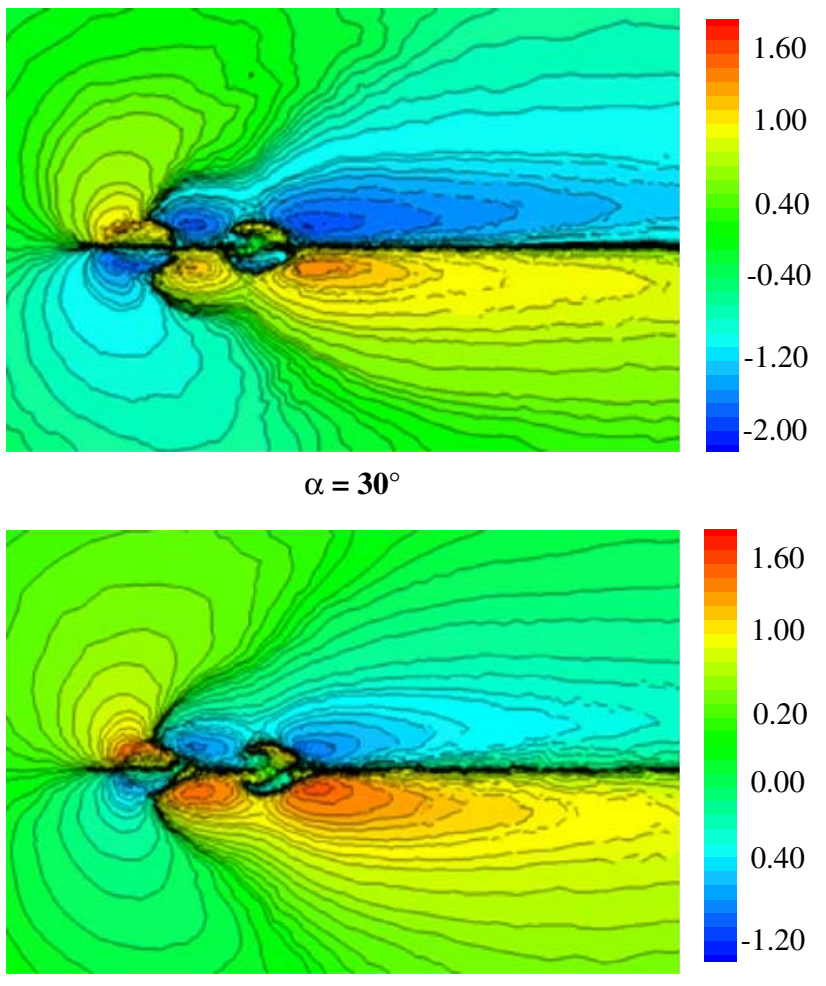

$\alpha=45^{\circ}$

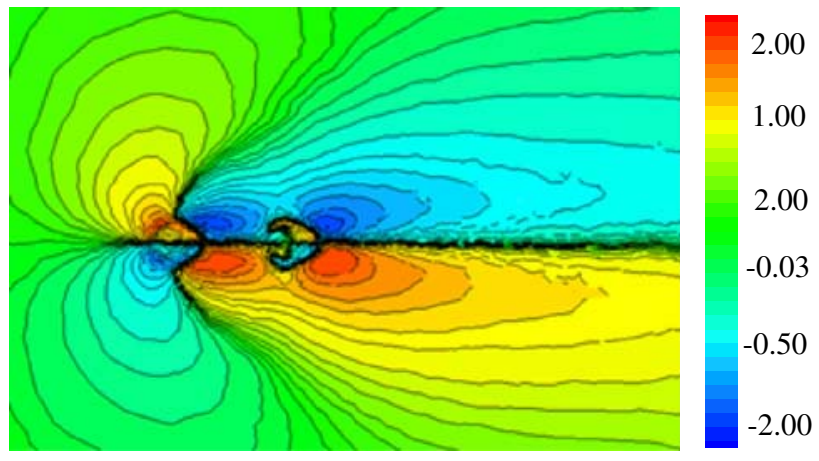

$\alpha=60^{\circ}$

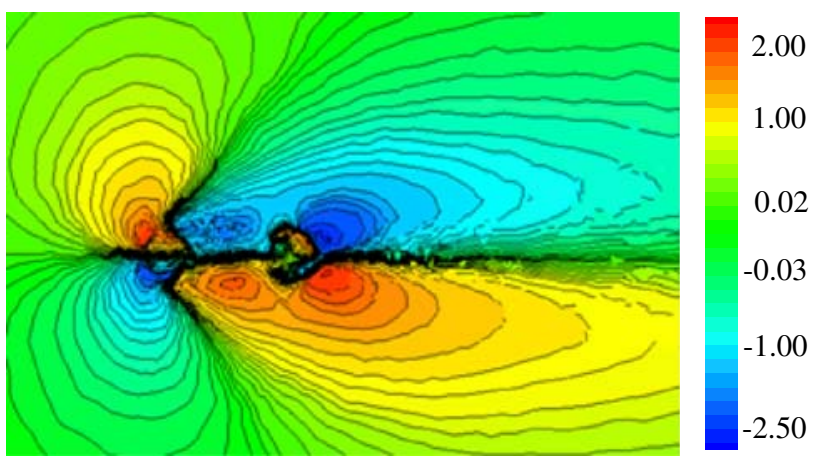

$\alpha=90^{\circ}$

Fig. 10 Spanwise velocity contours on the plane $y=2 \mathrm{~mm}$ under the different inclination cases 
Fig. 11 Spanwise velocity contours on two different vertical planes under the different inclination cases: a $y=5 \mathrm{~mm}$ and b $y=8 \mathrm{~mm}$ (a)

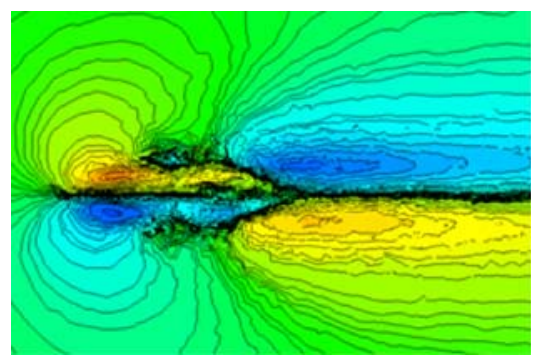

(b)
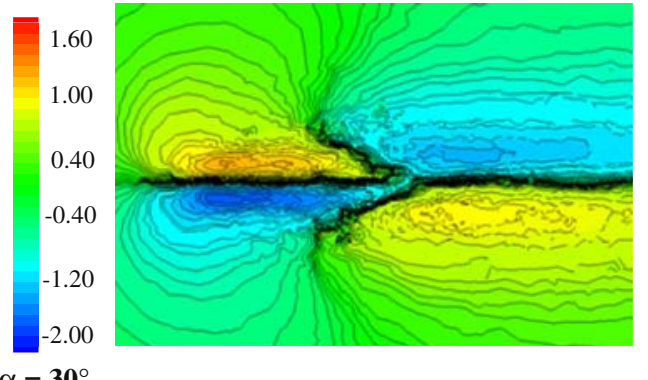

$\alpha=\mathbf{3 0}^{\circ}$
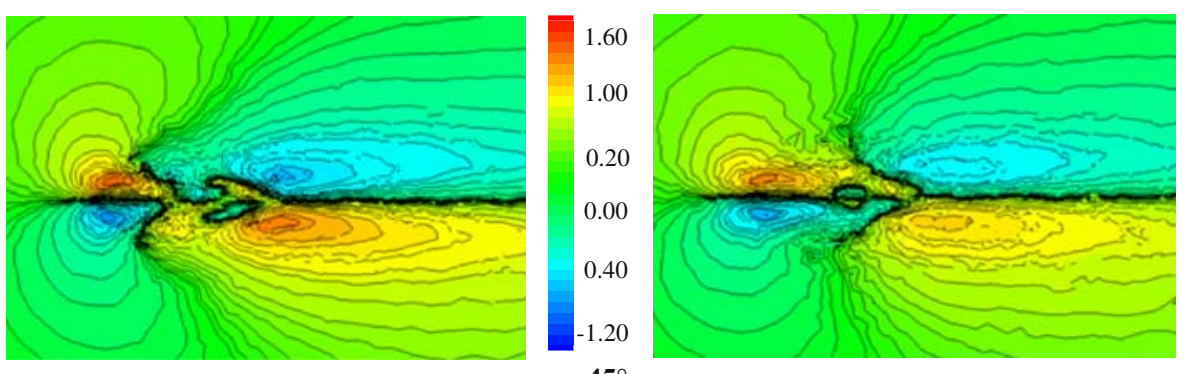

$\alpha=\mathbf{4 5}^{\circ}$
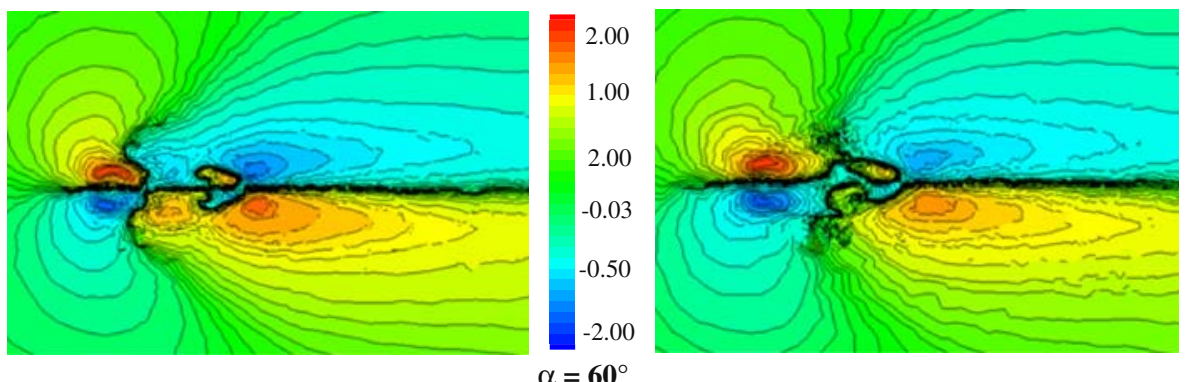

$\alpha=6^{\circ}$

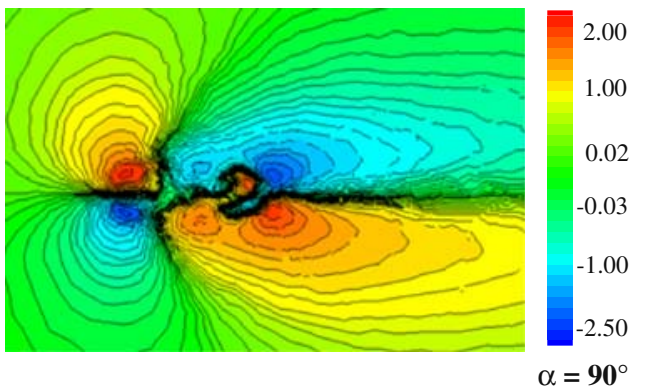

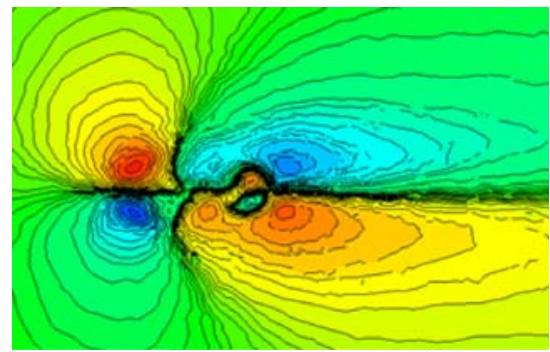

and begins affecting the third. This phenomenon is more explicitly explained with reference with Fig. 11 that represent the contours of the lateral velocity components, respectively, on the planes $y=5 \mathrm{~mm}$ (Fig. 11a) and $y=8 \mathrm{~mm}$ (Fig. 11b). On the intermediate plane $(y=5 \mathrm{~mm})$, we see that under the weakest inclination angle, the first jet has already joined the following one that is why we no longer detect the presence of the reverse flow region. When we finally attain the highest plane $(y=8 \mathrm{~mm})$, the joining of the twin jets is completely perceptible under the two-first cases and becomes to occur under the third one. Under the final case, the initial impulse brought by the inclination angle is still sufficient to not allow the joining of the jets; but if we go beyond this plane ( $y>8 \mathrm{~mm}$ ) we will certainly assist to this joining and then to the total vanishing of the periphery peaks.

When we move downstream of each of the jets; the upstream one and then both of them; we no longer detect their proper velocity magnitude but that of the reverse flow region contained between the jet nozzles and downstream of both of them (Fig. 12). That is why when we move from the rear jet location to the following region, the velocity distribution is totally inversed. Nevertheless, the twin peaks are preserved but no longer express the same physical phenomenon: in the current location they are evidence of the forming of the characteristic vortex pair that follows 
Fig. 12 Impact of the streamwise inclination on the lateral evolution of the spanwise velocity component between and downstream of the twin jets' nozzles

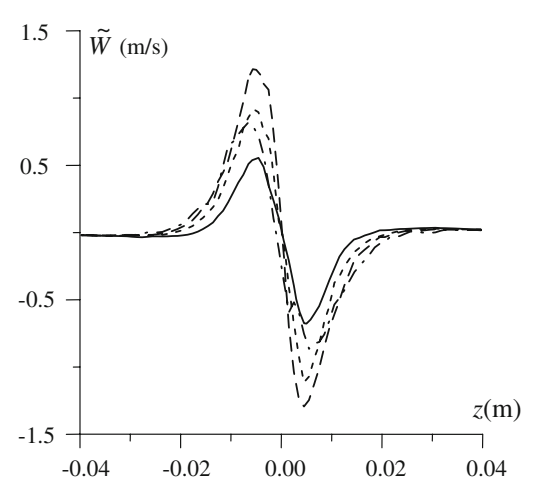

(a) $y=\mathbf{2} \mathrm{mm}$
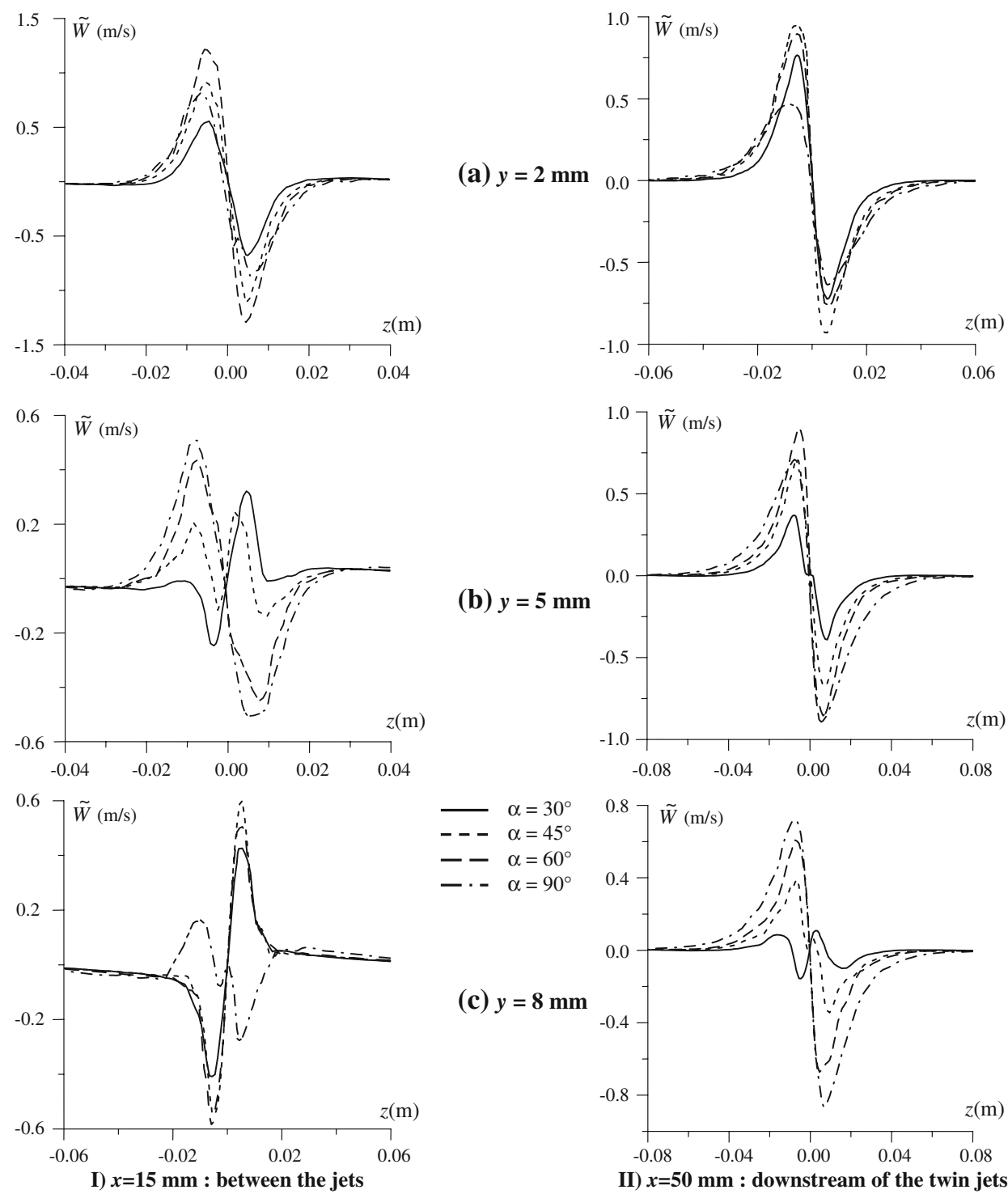

(b) $y=5 \mathrm{~mm}$

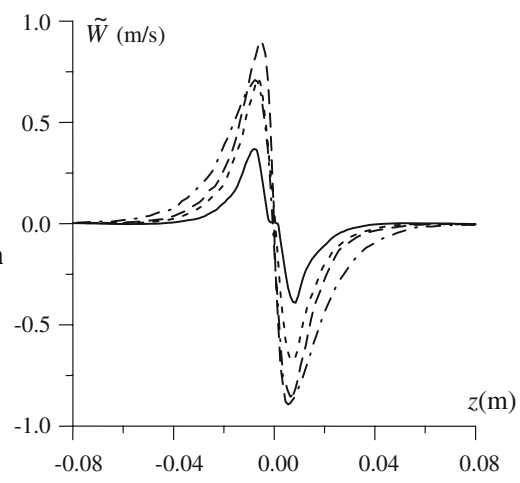

$\begin{array}{ll}- & \alpha=30^{\circ} \\ --- & \alpha=45^{\circ} \\ -- & \alpha=60^{\circ} \\ -- & \alpha=90^{\circ}\end{array}$

(c) $y=8 \mathrm{~mm}$

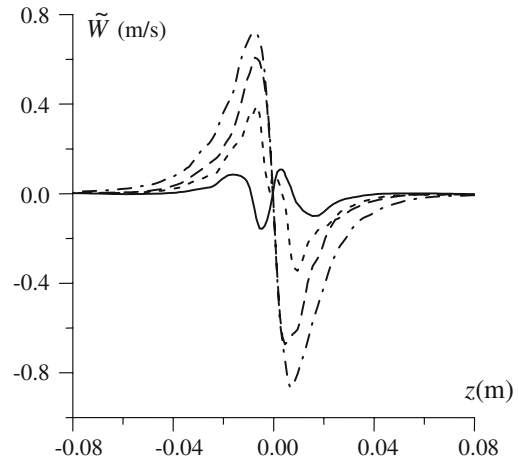

II) $x=50 \mathrm{~mm}:$ downstream of the twin jets the rear jet that balances out the wake region developed by the free stream around the jet. On the centerline $(z=0)$, the velocity is null suggesting that the slower moving elements of the crossing flow have a high probability of being entrained upward into the underside of the jet. As to the mean velocity values, they quantify the width of the wake zone that is more pronounced when the jets are more straightened. This higher width results in a slower decay rate as it entrains a more important mixing of the twin jets within the environing crossflow. Moving upward (increasing $y$ ) enables us to follow the jets when crossing the mainstream vertically and being distorted and flattened on the streamwise direction. The latter is completely established under the weakest inclination case $\left(30^{\circ}\right)$ since the jet has not enough "strength" to cross the mainstream; under the second one $\left(45^{\circ}\right)$ the expansion started to settle down but not sufficiently strong to completely inverse the velocity distribution. The rhythm at which this process takes place is then closely related to the initial inclination angle and to the "impulse" it provides to them. When we reach the farthest plane $(y=8 \mathrm{~mm})$, the velocity distribution has not been inversed yet; that is certainly due to the impulse that is still strong to allow the jets to be bent; but if we go beyond this plane we will certainly assist to it (it has already began but did not completely develop).

\subsection{Shear stress}

The choice of the RSM turbulent model is essential in our case is due to the complexity and the variety of the vortical system developed in the resulting flowfield. These vortices are generated from the double interaction of the twin emitted jets with the oncoming crossflow and with each others. Four main structures are established as mentioned 
Fig. 13 Vertical distribution of the $\overline{u^{\prime \prime} u^{\prime \prime}}$-normal stress within the different characterizing streamwise locations and on the symmetry plane $(z=0)$

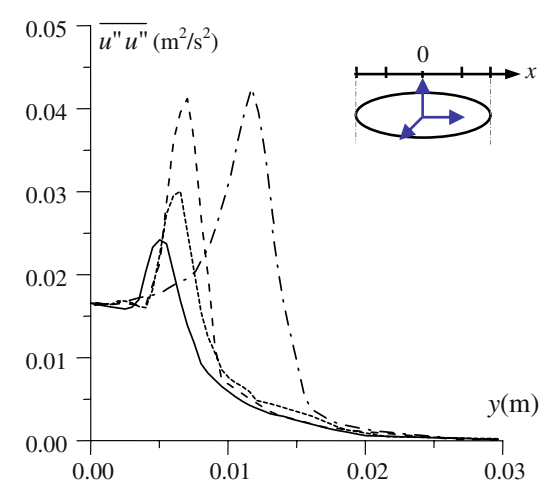

(a) $x=0 \mathrm{~mm}$
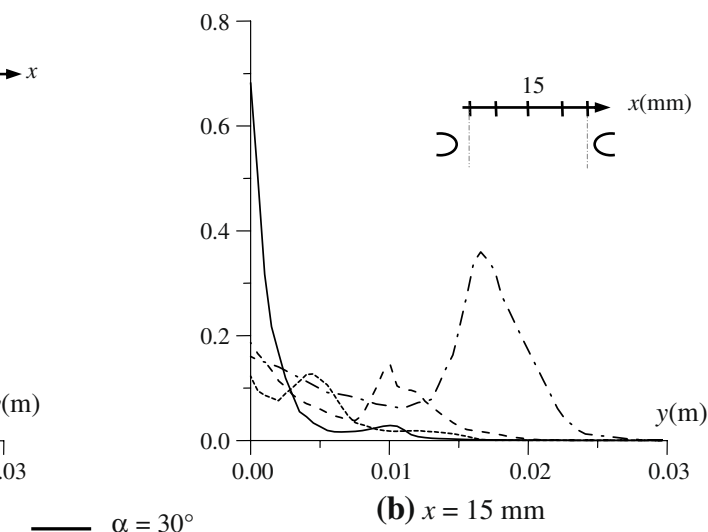

(b) $x=15 \mathrm{~mm}$

$--\alpha=45^{\circ}$

$--\alpha=60^{\circ}$

- - $\alpha=90^{\circ}$

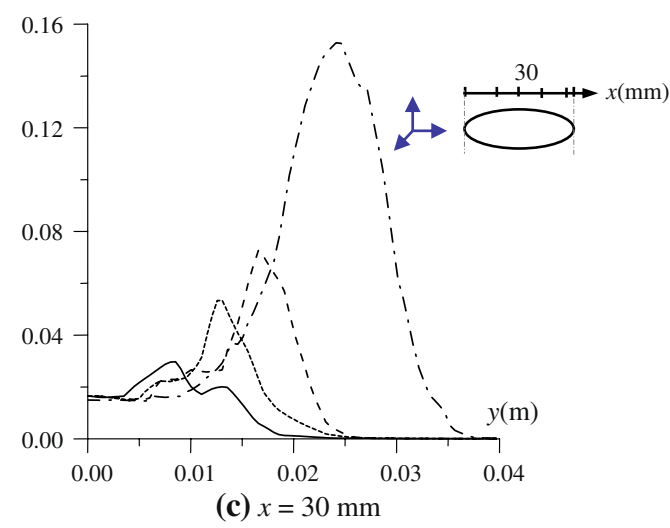

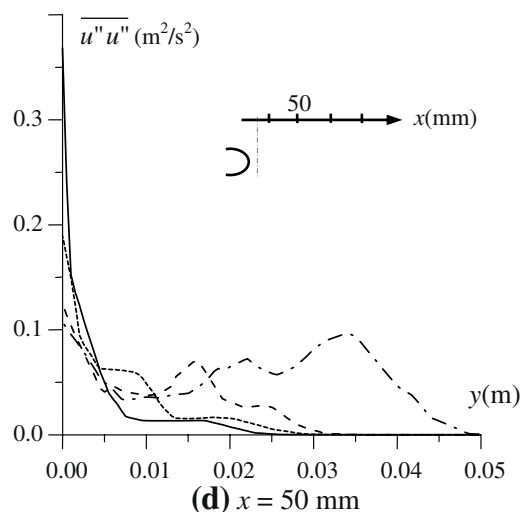

before and the distribution of the Reynolds stresses is the best way to scale and evaluate them. For the matter we propose to consider first the vertical distribution of the normal $\overline{u^{\prime \prime} u^{\prime \prime}}$ stress within the different characterizing regions of the domain in Fig. 13.

A global sight to the different distributions reveals, before any deep examination, that the more distinct stress peaks are depicted within the twin jets' columns; which does not automatically mean higher stress values. This region is in fact an interaction zone between the twin jets themselves and between the jets and the oncoming crossflow. If the $\overline{u^{\prime \prime} u^{\prime \prime}}$ stress attains its maximum in this zone it is simply because it shields a high interaction activity indicating a maximum turbulence and subsequent diffusion production and. We propose to examine more closely all these variations and to begin let us consider the first jet location (Fig. 13a) where all the profiles share the same initial value that is contained in the first jet "background". Once the upstream jet is emitted, we assist to the establishment of a single peak that is attained farther as the jets are straightened and whose magnitude goes increasing with the initial inclination angle. This increasing is logic since straightening the jets provides them with a higher impulse allowing them to cross the oncoming mainstream deeper before undergoing its deflecting process. The development of this peak is then simply generated by the interaction of the rear jet with the crossflow and its resistance to the deflection the latter experiences on it. Within the downstream jet location (Fig. 13c), the peaks are rather registered farther from the injection plate $(y>10 \mathrm{~mm})$. In fact, due to the shielding effect of the rear jet, the second one is likely to progress deeper in the domain before being deflected. The turbulent activity which is engendered by the interaction of the emitted jets and the environing flow is consequently delayed which justifies the later peaks registration. It is further delayed as the jets are straightened due to their farther emission within the domain (increasing $y$ coordinates).

Between and far downstream of the jets locations, we note a further interesting phenomenon. It consists in the registration of a maximum initial value under the weakest inclination case. This may be explained by the fact that under this case; the rear jet is kept close to the injection plate which allows it to exert an important perturbation on it. Elsewhere, we find back same order peaks: the highest and farthest peak of the $\overline{u^{\prime \prime} u^{\prime \prime}}$ stress is attained under the 
Fig. 14 Vertical distribution of the $\overline{v^{\prime \prime} v^{\prime \prime}}$-normal stress within the different characterizing streamwise locations and on the symmetry plane $(z=0)$

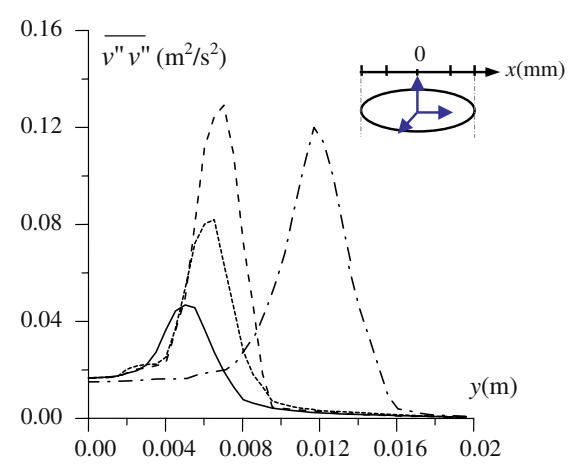

(a) $x=0 \mathrm{~mm}$

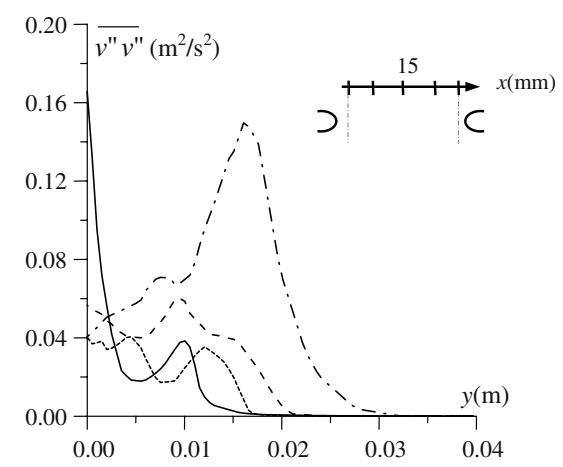

b) $x=15 \mathrm{~mm}$

$$
\begin{aligned}
& -\alpha=45^{\circ} \\
& --\alpha=60^{\circ} \\
& --\alpha=90^{\circ}
\end{aligned}
$$

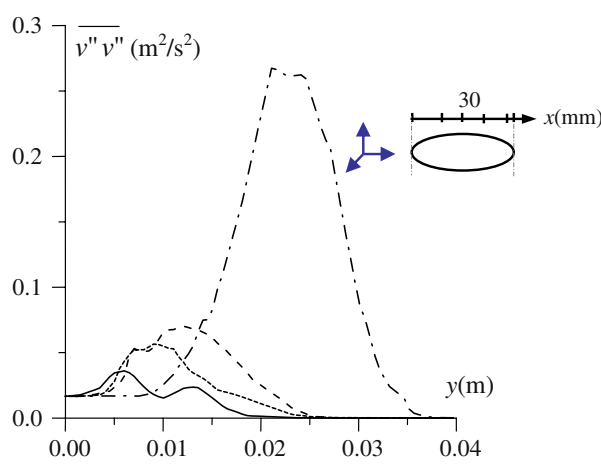

(c) $x=30 \mathrm{~mm}$

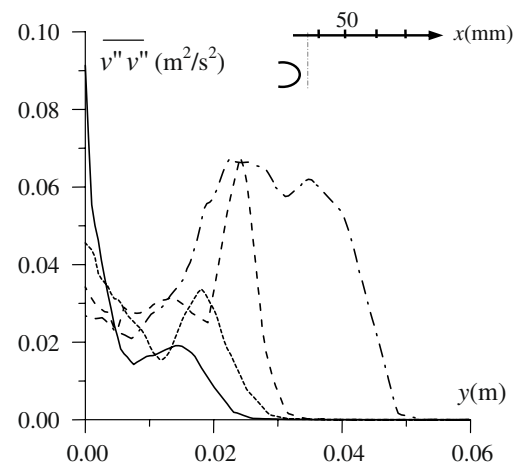

(d) $x=50 \mathrm{~mm}$ highest inclination angle. When we reach the second jet location, we find back the same behavior adopted within the rear jet with, however, a global augmentation of the attained peaks; the initial adopted value being kept identical. This augmentation is due to our position within the interaction zone; nevertheless it is not as important as between the jets' columns because the second jet does not entirely participate in this interaction since it is "protected" by the rear one. The global vanishing occurs, however, later because when we move downstream, the interaction takes place higher: after the jets' lifting. Finally, when we go beyond of the twin jet locations (Fig. 13d), the normal $\overline{u^{\prime \prime} u^{\prime \prime}}$ stress rises once again but attains less important peak values: in this zone we are no longer in the first and direct stage of the interaction. We are rather within in presence of stress that originates from small scale ring like vortices that still exist on the windward side of the combined jets.

In addition to the $\overline{u^{\prime \prime} u^{\prime \prime}}$ normal stress, we propose to plot the vertical distribution of the $\overline{v^{\prime \prime} v^{\prime \prime}}$ normal stress on Fig. 14. The present feature adopts a similar behavior as the $\overline{u^{\prime \prime} u^{\prime \prime}}$ component; a significant difference is, however, perceived on their scales. In fact, whereas the $\overline{u^{\prime \prime} u^{\prime \prime}}$ stress attains its maximum between the twin jets' locations, the $\overline{v^{\prime \prime} v^{\prime \prime}}$ stress component reaches its maximum within the downstream jet location. The second discrepancy consists in attaining the minimum value of the stress downstream of the twin jets (Fig. 14d) which is not the case for the previously considered stress component since the latter still had a significant value in this region. The registration of these maximum values informs actually about our location within the domain since it corresponds to our crossing of the counter rotating vortex par as stated by Tyagi and Acharya [29]. This statement is true whatever the scale of the attained peaks which brings further importance on its significance.

Figure 15 represents the vertical distribution of the $\overline{u^{\prime \prime} v^{\prime \prime}}$ streamwise stress component where two major behaviors develop: within the jets' locations the $\overline{u^{\prime \prime} v^{\prime \prime}}$ stress varies within a large interval containing positive and negative values; whereas between and beyond the jets this strain is initially and shortly negative to finally be contained within a small interval neighboring zero. Let us come back to what happens within the jets' nozzles to examine the influence of the initial inclination angle on this particular feature. Within the first jet location one for example, we see that the under the weakest inclination angle the $\overline{u^{\prime \prime} v^{\prime \prime}}$ stress attains a single peak before vanishing. The augmentation of this inclination parameter reduces the 
Fig. 15 Vertical distribution of the $\overline{u^{\prime \prime} v^{\prime \prime}}$ — stress within the different characterizing streamwise locations and on the symmetry plane $(z=0)$

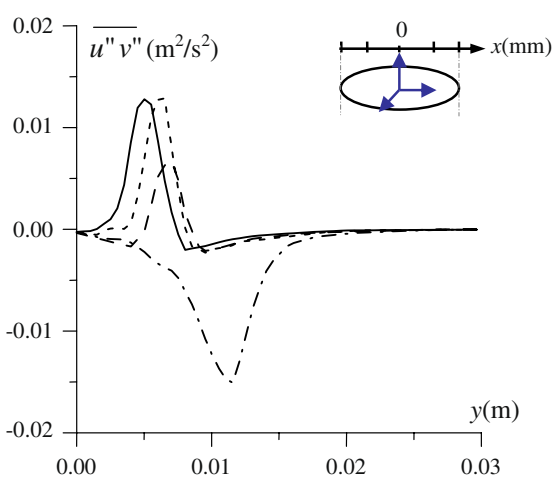

(a) $x=0 \mathrm{~mm}$

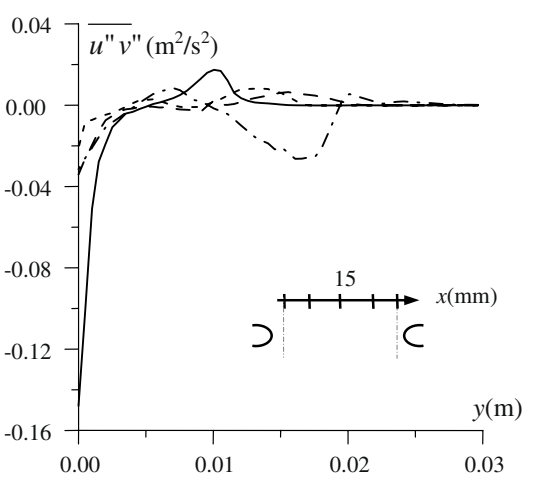

(b) $x=15 \mathrm{~mm}$

$--\alpha=45^{\circ}$

$-\alpha=60^{\circ}$

- - $\alpha=90^{\circ}$

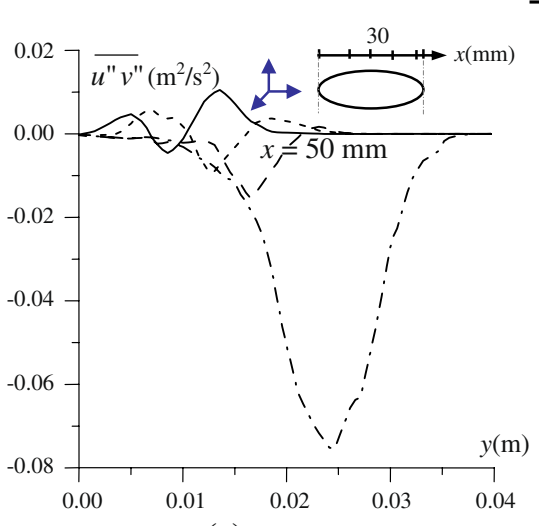

(c) $x=30 \mathrm{~mm}$

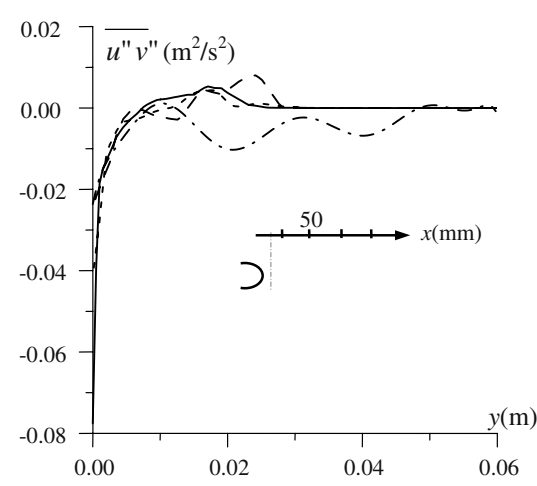

(d) $x=50 \mathrm{~mm}$ registered peak and succeeds even to inverse its value since under $\alpha=90^{\circ}$ (Fig. 15a) the stress is overall negative by attaining a deep minimum before completely vanishing. The $\overline{u^{\prime \prime} v^{\prime \prime}}$ stress is stated by Tyagi and Acharya [29] to express the jets penetration within the environing crossflow as well as the vertical mixing rate; thus the positive attained peaks are probably expressing the high mixing rate attained at the interface between the rear jet and the oncoming crossflow. As to the negative peak, it would express the very weak mixing occurring within the first jet location since under the highest inclination angle the jet is first emitted farther from the injection plate and that is farther that it accuses a significant deflection. The transition towards the downstream jet deepens this negative peak before vanishing and the reached negative peak is actually an evidence of our passage through the lee-side of the expansion of the rear jet. The same trend is adopted by the profile relative to $\alpha=60^{\circ}$ with, however, a shallower peak. Under the remaining and weaker inclination cases two further positive peaks are registered before vanishing and are probably due to the near position to the wall.

Between the jets' nozzles and far downstream, the $\overline{u^{\prime \prime} v^{\prime \prime}}$ stress starts from a negative value that is deeper for the weakest inclination angle. This reduced initial value is essentially due to our progression from the lee-side of the rear jet and the combination of the twin jets, respectively. However, this parameter rapidly increases to remain in the vicinity of the zero along the rest of the domain even if some variation is present and is relatively more pronounced under the highest inclination angle.

\section{Conclusion}

The present dynamic study of two inline jets emitted within a cooler oncoming crossflow revealed the presence of a complex resulting flowfield. This complexity is expressed by the establishment of an elaborated vortical system composed of four main vortices: the horseshoe vortices, the upright vortices, the shear layer vortices and the counter rotating vortex pair. They are essentially due to momentum and pressure gradients; nevertheless their origin and exact mechanism are still not well established. This study demonstrated the extremely important role of the initial inclination angle of the emitted jets in their mutual interaction as well in their mixing within the mainstream. In fact, 
increasing the initial emission angle provides the jets with a higher "impulse" to expand deeper vertically in the environing flow which results in a further vertical mixing. Decreasing the initial inclination factor, on the contrary, allows rather a spanwise expanding since the jets are rapidly deflected by the oncoming crossflow; which promotes the trapping of the jets' flow close to the injection plate. This eventuality is largely preferred in the case we handle polluted jets that we want to control rather than spread in the atmosphere (or water in water for liquid jets). All these conclusions were drawn from the experimentally visualized data by means of the PIV and the numerically processed results by means of the finite volume method and the RSM second-order turbulent closure model. Introducing the latter in such a configuration counts for the new brand brought by our work and presents the advantages of processing the different shear stresses and detecting the slightest turbulent variations that are responsible for the different developed vortices. We also examined the impact of the initial inclination on the shear stress components and could show that the straightest are the jets, the highest are the shear stresses. This could be justified by the fact that the straightest jets are provided with a stronger "impulse" that engenders a more "violent" interaction with the mainstream and then results in stronger vortices and consequently in a better mixing which is not a good idea in case of contaminated jets.

Open Access This article is distributed under the terms of the Creative Commons Attribution Noncommercial License which permits any noncommercial use, distribution, and reproduction in any medium, provided the original author(s) and source are credited.

\section{References}

1. Ohanian T, Rahai HR (2001) Numerical investigations of multi turbulent jets in a crossflow. 39th AIAA Aerospace Science Meetings and Exhibit, Reno, Nevada, Paper No AIAA2001-1049, January 2001

2. Disimile PJ, Dimicco RG, Toy N, Savory E (1990) The development of twin jets issuing into a crossflow. 12th Symposium on Turbulence. University of Missouri-Rolla Rolla, MO, 24-26 September 1990

3. Makihata T, Miyai Y (1979) Trajectories of single and double jets injected into a crossflow of arbitrary velocity distribution. J Fluids Eng 101:217-223

4. Ibrahim IM, Gutmark EJ (2006) Dynamics of single and twin circular jets in crossflow. 44th AIAA Aerospace Sciences Meeting and Exhibit. Reno, Nevada, Paper No. AIAA2006-1281, 9-12 January 2006

5. Ziegler H, Wooler PT (1973) Analysis of stratified and closely spaced jets exhausting into a crossflow. National Aeronautics and space Administration, Washington, DC. November 1973

6. Ziegler H, Wooler PT (1971) Multiple jets exhausted into a crossflow. J Aircraft 8(6):414-420

7. Isaac KM, Jakubowski AK (1985) Experimental study of the interaction of multiple jets with a cross flow. AIAA J 23(11): $1679-1683$
8. Xiao D (1992) Experimental and computational investigation of multiple jets in a duct crossflow. Ph.D. thesis, Department of Chemical Engineering, Queen's University, Kingston

9. Maidi M, Yao Y (2008) Numerical visualization of vortex flow behavior in square jets in cross-flow. J Vis 11(4):319-327

10. Radhouane A, Mahjoub Saïd N, Mhiri H, Le Palec G, Bournot Ph (2009) Impact of the initial streamwise inclination of a double jet emitted within a cool crossflow on its temperature field and pollutants dispersion. J Heat Mass Transf 45(6):805-816

11. Andreopoulos J, Rodi W (1984) Experimental investigation of jets in a crossflow. J Fluid Mech 138:93-127

12. Baker CJ (1979) The laminar horseshoe vortex. J Fluid Mech 95(2):347-367

13. Mariotti V, Camarri S, Savetti MV, Koobus B, Dervieux A, Guillard H, Wornom S (2005) Numerical simulation of a jet in crossflow, RR N`5638, Juillet 2005

14. Kelso RM, Lim TT, Perry AE (1996) An experimental study of round jets in cross-flow. J Fluid Mech 306:111-144

15. Fric TF, Roshko A (1994) Vortical structure in the wake of a transverse jet. J Fluid Mech 279:1-47

16. Kamotani Y, Greber I (1972) Experiments on a turbulent jet in a cross flow. AIAA J 10:1425-1429

17. Cortelezzi L, Karagozian AR (2001) On the formation of the counter-rotating vortex pair in transverse jets. J Fluid Mech 446:347-373

18. Lim TT, New TH, Luo SC (2001) On the development of largescale structures of a jet normal to a cross flow. Phys Fluids 13(3):770-775

19. Rivero A, Ferre JA, Giralt F (2001) Organized motions in a jet in crossflow. J Fluid Mech 444:117-149

20. Smith SH, Mungal MG (1998) Mixing, structure and scaling of the jet in crossflow. J Fluid Mech 357:83-122

21. Muppidi S, Mahesh K (2006) Two-dimensional model problem to explain counter-rotating vortex pair formation in a transverse jet. Phys Fluids J 18:85-103

22. Kolar V, Takao H, Todorok T, Savory E, Okamoto S, Toy N, (2001) Vorticity transport associated with the dominant vortical structures of twin jets in crossflow. 14th Australasian Fluid Mechanics Conference, Adelaide University, Adelaide, Australia, 10-14 December 2001

23. Kolar V, Takao H, Todoroki T, Savory E, Okamoto S, Toy N (2003) Vorticity transport within twin jets in crossflow. Exp Therm Fluid Sci 27:563-571

24. Kolar V, Savory E, Takao H, Todoroki T, Okamoto S, Toy N (2006) Vorticity and circulation aspects of twin jets in cross-flow for an oblique nozzle arrangement. Proc Inst Mech Eng Part G J Aerosp Eng 220(4):247-252

25. Kolar V, Savory E (2007) Dominant flow features of twin jets and plumes in crossflow. J Wind Eng Ind Aerodyn 95:1199-1215

26. Ibrahim IM (2006) An experimental study of single and twin transverse jets in subsonic. Thesis submitted to the division of research and advanced studies of the University of Cincinnati, in partial fulfillment of the requirements for the degree of Master of Science, March 2006

27. Patankar SV, Spalding DB (1972) A calculation procedure for heat, mass and momentum transfer in three dimensional parabolic flows. Int J Heat Mass Transf 15:1787-1806

28. Demuren AO, Rodi W (1987) Three dimensional numerical calculations of flow and plume spreading past cooling towers. J Heat Transf 109:113-119

29. Tyagi M, Acharya S (1999) Large Eddy simulations of jets in crossflow: effect of free-stream turbulence intensity. Proceedings of the ASME/JSME fluid engineering meeting, San Francisco, ASME-FEDSM99-7799, July 1999 